\title{
Les aménagements agricoles dans les Marges arides de Syrie du Nord $\left(5^{e_{-1}} \mathrm{O}^{\mathrm{e}}\right.$ siècles $)$
}

Des témoins des modalités d'appropriation et d'exploitation des campagnes

\author{
Marion Rivoal et Marie-Odile Rousset
}

La plus grande partie de la région étudiée ici se situe à l'est d'une ligne Damas-Alep, dans la province de Hama, et correspond à une zone désormais connue en Syrie sous le nom de «Marges arides du Croissant fertile» ou encore «Marges arides de la Syrie du Nord » ${ }^{1}$. Elle correspond au territoire de la cité de Chalcis du Bélus, fortement hellénisée, devenue Qinnasrin à l'époque islamique $^{2}$. Les résultats présentés ici sont issus d'enquêtes de terrain sur une zone pour laquelle nous ne disposons pas de textes mais seulement de quelques études épigraphiques. Seuls de très rares sites ont été fouillés et les autres vestiges archéologiques n'ont été envisagés que sous l'angle de la prospection. Cependant, leur état de conservation exceptionnel en fait une source matérielle incontournable pour l' analyse de la politique économique agricole en Syrie du Nord entre le $5^{\mathrm{e}}$ et le $10^{\mathrm{e}}$ siècle. Les vestiges agraires (épierrements, parcellaires, enclos), les grands ouvrages hydrauliques pour l'irrigation et l'alimentation en eau des hommes et des animaux d'élevage témoignent

1 Voir par exemple Bernard Geyer, éd., Conquête de la steppe et appropriation des terres sur les marges du Croissant fertile (Lyon: Maison de l' Orient et de la Méditerranée, 2001); Ronald Jaubert et Bernard Geyer, éds, Les marges arides du Croissant fertile: peuplements, exploitation et contrôle des ressources en Syrie du Nord (Lyon: Maison de l'Orient et de la Méditerranée, 2006); Pierre-Louis Gatier, Bernard Geyer et Marie-Odile Rousset, éds, Entre nomades et sédentaires: prospections en Syrie du Nord et en Jordanie du Sud (Lyon: Maison de l'Orient et de la Méditerranée, 2010) et Marie-Odile Rousset, Bernard Geyer, Pierre-Louis Gatier et Nazir Awad, Habitat et environnement: prospections dans les marges arides de la Syrie du Nord, Conquête de la steppe (Lyon: Maison de l'Orient et de la Méditerranée, 2016), 9-17 pour l'ensemble de la bibliographie sur la région.

2 Pierre-Louis Gatier, “«Grande» ou «petite Syrie Seconde»? Pour une géographie historique de la Syrie intérieure protobyzantine," dans Conquête de la steppe et appropriation des terres sur les marges du croissant fertile, éd. Bernard Geyer (Lyon: Maison de l' Orient et de la Méditerranée, 2001), 105. Sur les recherches archéologiques récentes conduites sur ce site, voir Marie-Odile Rousset, "De Chalcis à Qinnasrin," dans Topoi: villes et campagnes aux rives de la Méditerranée ancienne: hommages à Georges Tate, éds. Gérard Charpentier et Vincent Puech, Supplément 12 (2013), 311-340. 
d'une prise en compte de l' ensemble des potentiels agricoles et d'une organisation territoriale centralisée.

Pour les besoins de cet article, nous avons choisi d'étendre la zone prise en considération à l'ensemble des trois plateaux basaltiques - les Jabal al-'Alā, al-Ḥaș et Shbayț - qui bordent, au nord et à l'ouest, la steppe aride. Chacun d'entre eux fournit en effet des indications importantes sur les modes de gestion de ces campagnes pendant l'Antiquité tardive ${ }^{3}$.

La région dans son ensemble, avec une dotation pluviométrique modique (entre $150 \mathrm{~mm}$ et $400 \mathrm{~mm}$ de précipitations moyennes annuelles) et très variable d'une année à l'autre ${ }^{4}$, est par définition une zone de marge. Les stratégies de mise en valeur, quelles qu'elles soient (culture, élevage, association des deux), y sont nécessairement soumises à l'aléatoire ${ }^{5}$. Les Marges

3 À la zone prospectée au cours du programme «Marges arides» s' ajoutent ainsi l'ensemble du Jabal al-'Alā, pour lequel on possède des données bibliographiques importantes (Jean Lassus, Inventaire archéologique de la région au nord-est de Hama, 2 vols. (Damas: Institut français et Paris: Leroux, 1935-1936); Howard Crosby Butler, Syria, Publications of the Princeton University Archaeological Expeditions to Syria in 1904-1905 and 1909, vol. 2, Architecture, Section B, Northern Syria (Leyde: Brill, 1920)), ainsi que le Jabal al-Has et le Jabal Shbayț, pour lesquels la bibliographie, ancienne ou relativement ancienne (Howard Crosby Butler, Syria: Publications of an American Archaeological Expedition to Syria in 1899-19oo, vol. 2, Architecture and Other Arts (New York: the Century Company, 1903); René Mouterde et Antoine Poidebard, Le Limes de Chalcis: organisation de la steppe en Haute-Syrie romaine, 2 vols. (Paris: Geuthner, 1945); Heinz Gaube, "Die syrischen Wüstenschlösser: einige wirtschaftliche und politische Gesichtspunkte zu ihrer Entstehung," Zeitschrift des Deutschen Palästina-Vereins 95 (1979): 195-209; Claus-Peter Haase, "Ein archäologischer Survey im Gabal Sbet und im Gabal al-Ahass," Damaszener Mitteilungen 1 (1983): 69-76), a pu être complétée ces dernières années par des prospections (Jean-Baptiste Rigot, "Environnement naturel et occupation du sol dans le bassin-versant du lac Jabbûl (Syrie du Nord) à l'Holocène” (PhD diss., Université Lumière-Lyon 2, 2003); Marion Rivoal, "Le peuplement byzantin et la mise en valeur de la Syrie centrale: l' exemple des plateaux basaltiques (Jebel al-'Ala, Jebel Hass et Jebel Shbayt)," dans Regards croisés sur l'étude archéologique des paysages anciens: nouvelles recherches dans le Bassin méditerranée, en Asie centrale et au Proche et Moyen-Orient, éds. Hala Alarashi et al. (Lyon: Maison de l'Orient et de la Méditerranée, 2010), 79-92; Marion Rivoal, "Le peuplement byzantin des massifs basaltiques de la Syrie centrale: modalités d'occupation du sol et stratégies de mise en valeur," dans Entre nomades et sédentaires. Prospections en Syrie du Nord et en Jordanie du Sud, éds. Pierre-Louis Gatier, Bernard Geyer et Marie-Odile Rousset (Lyon: Maison de l'Orient et de la Méditerranée, 2010), 209-240; Marion Rivoal, "Entre steppe et plateaux basaltiques: l' occupation de la Syrie centrale à la période byzantine," Syria $88(2011))$.

4 Voir Myriam Traboulsi, "Les précipitations dans les Marges arides de la Syrie du Nord," dans Entre nomades et sédentaires. Prospections en Syrie du Nord et en Jordanie du Sud, éds. PierreLouis Gatier, Bernard Geyer et Marie-Odile Rousset (Lyon: Maison de l'Orient et de la Méditerranée, 2010), 73-108.

5 Sur cette "culture de l' aléatoire," voir Jean Métral, “Économie et sociétés: stratégies alterna- 
arides de la Syrie du Nord rassemblent des milieux contrastés, souvent profondément imbriqués ${ }^{6}$, où se côtoient traditionnellement des populations de cultivateurs-éleveurs sédentaires et de pasteurs nomades ou semi-nomades. Aussi, lorsqu' on évoque une «ruée vers l'est», un processus de conquête ou encore une tentative d'appropriation des territoires situés à l'est de l'axe Hama-Alep, choisit-on implicitement le point de vue des sédentaires. Les zones à l' est de l' isohyète des $200 \mathrm{~mm}$ - seuil théorique de l' agriculture pluviale -, majoritairement délaissées par les sédentaires, hormis au cours de l'âge du Bronze $^{7}$, constituaient les terrains de parcours des pasteurs nomades, dans l'Antiquité comme jusqu' au milieu du $19^{\mathrm{e}}$ siècle (bādiya), période durant laquelle commence le repeuplement contemporain de ces régions. Du $5^{\mathrm{e}}$ au $7^{\mathrm{e}}$ siècle de notre ère, ces zones de pâture paraissent cependant en grande partie sinon en totalité - avoir été acquises aux sédentaires. C' est ce qu' indiquent le semis serré des sites de ces périodes et la densité des aménagements hydroagricoles qui les accompagnent. Ces installations, avec leurs spécificités, renvoient à un système de mise en valeur fondé sur les potentiels agronomiques des différents milieux ${ }^{8}$, particulièrement à la fin de la période byzantine. Les productions régionales, diversifiées, sont manifestement intégrées dans des réseaux d'échanges régionaux voire extra-régionaux ${ }^{9}$. Ce phénomène semble $s^{\prime}$ intensifier aux $7^{\mathrm{e}}-8^{\mathrm{e}}$ siècles puisque, malgré la diminution du nombre des sites à cette époque, les monnaies omeyyades retrouvées en prospection sont proportionnellement beaucoup plus nombreuses que les monnaies byzantines $^{10}$.

tives et cultures de l'aléatoire," dans Steppes d'Arabie: états, pasteurs, agriculteurs et commerçants: le devenir des zones sèches, éds. Riccardo Bocco, Ronald Jaubert et Françoise Métral (Paris: Presses universitaires de France et Genève: Cahiers de l'I.U.E.D.), 385-386. Jacques Besançon et Bernard Geyer, "Contraintes écogéographiques et modes d'occupation du sol," dans Les marges arides du croissant fertile: peuplements, exploitation et contrôle des ressources en Syrie du Nord, éds. Ronald Jaubert et Bernard Geyer (Lyon: Maison de l'Orient et de la Méditerranée, 2006), 11-53.

7 Bernard Geyer, Jacques Besançon et Marie-Odile Rousset, "Les peuplements anciens," dans Les marges arides du croissant fertile, éds. Ronald Jaubert et Bernard Geyer (Lyon: Maison de l'Orient et de la Méditerranée, 2006), 55-6o.

8 Ronald Jaubert et al., éds, Land Use and Vegetation Cover in the Semi-arid and Arid Areas of Aleppo and Hama Provinces (Syria) (Lyon: Maison de l' Orient et de la Méditerranée, 1999).

9 Rivoal, "Le peuplement byzantin des massifs basaltiques," 230-233; Rivoal, "Entre steppe et plateaux basaltiques," 161-163.

10 Olivier Callot, "Monnaies trouvées dans les Marges arides (Syrie)," dans Entre nomades et sédentaires: prospections en Syrie du Nord et en Jordanie du Sud, éds. Pierre-Louis Gatier, Bernard Geyer et Marie-Odile Rousset (Lyon: Maison de l'Orient et de la Méditerranée, 2010), 271-288. Par comparaison avec ce qui a été observé ailleurs en Méditerranée 
Les vestiges matériels qu' ont laissés ces économies traduisent simultanément une forme de revendication du territoire et l'usage qui est fait par la suite de ce terroir, c'est-à-dire ses modalités d'exploitation. Les limites des champs, les aménagements destinés à assurer les rendements des cultures les plus fragiles et/ou les plus rentables ou bien à les augmenter de manière significative témoignent de formes d'appropriation et de contrôle des campagnes par les cultivateurs sédentaires qui évoluent entre le $5^{\mathrm{e}}$ et le $10^{\mathrm{e}}$ siècle. Il est possible aujourd'hui de suivre certaines des adaptations que subissent les installations hydro-agricoles, même si l'on manque encore d'éléments indispensables - notamment chronologiques - pour étayer nos discours. Ces évolutions, qui traduisent des changements dans les stratégies économiques adoptées par les populations, apportent indirectement des indications sur les modalités d' appropriation et de gestion des campagnes.

\section{Les vestiges agraires: des témoins de l'appropriation des campagnes}

Dans l' Antiquité, sur des terres jusque-là principalement exploitées pour leurs ressources pastorales, la mainmise des populations d'agriculteurs sédentaires s'est traduite par des aménagements agricoles de nature et de fonctions variées ${ }^{11}$. Seuls quelques exemples, parmi les étapes plus représentatives et les plus éclairantes de ce processus de formation des terroirs, seront abordés ici.

Les aléas de la conservation des vestiges agraires - et une pierrosité des sols plus élevée qu' ailleurs qui a favorisé, dans ces secteurs, leur fossilisation - nous conduisent à privilégier, pour ces exemples, les plateaux basaltiques de l' ouest et du nord de la région (figure 6.1).

La zone de steppe qui s' étire entre ces mesas et le bastion montagneux nordpalmyrénien (Jabal Bal'as), au sud et à l'est, n'est pas dépourvue d'aménage-

orientale, on pouvait s' attendre à une quantité de monnaies byzantines beaucoup plus importante que celle effectivement retrouvée. Mais ces résultats sont peut-être dus aux aléas de la prospection. Le petit nombre de monnaies de l'époque abbasside (trois, au maximum) est le reflet de la forte baisse du monnayage mise en évidence ailleurs en Syrie. Voir également Cécile Morrisson, "Le monnayage omeyyade et l'histoire administrative et économique de la Syrie," dans La Syrie de Byzance à l'Islam VII ${ }^{e}-V I I I^{e}$ siècles: Actes du Colloque International, Lyon-Paris, 11-15 septembre 199o, éds. Pierre Canivet et Jean-Paul Rey-Coquais (Damas: Institut Français d'Études Arabes de Damas, 1992), 309318.

11 L' "agriculture" est ici prise dans son sens le plus large, qui rassemble les activités de culture et d'élevage. 


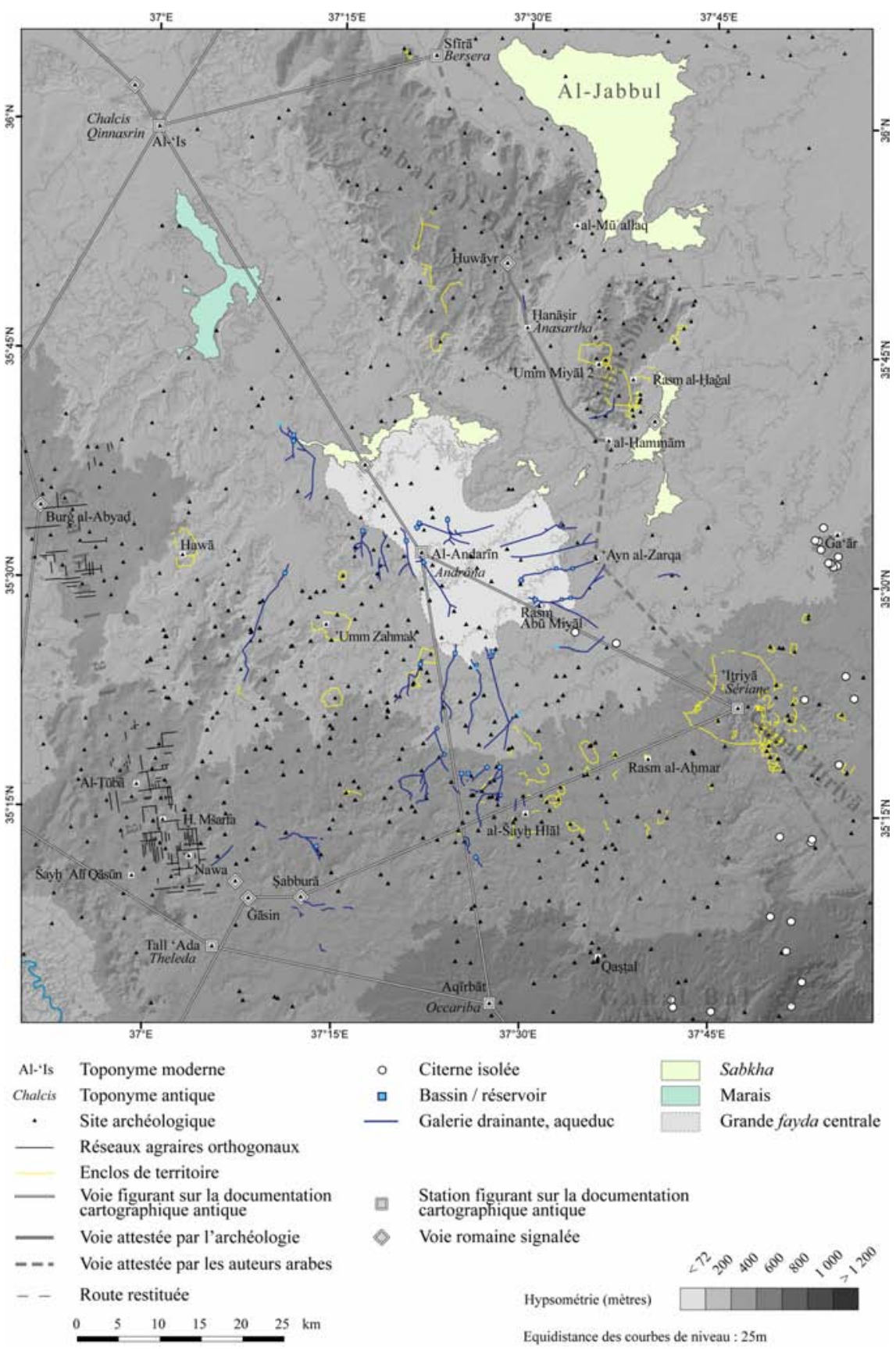

FIGURE 6.1 Carte générale de la région des Marges arides et des massifs basaltiques MARION RIVOAL, MARIE-ODILE ROUSSET, OLIVIER BARGE CMISSION MARGES ARIDES 2011 
ments agricoles - nous les évoquerons -, mais les efforts des populations d' agriculteurs pour constituer et organiser un terroir n' apparaissent nulle part plus clairement que dans les secteurs basaltiques ${ }^{12}$.

\subsection{L'épierrement}

Dans le contexte particulier des plateaux basaltiques (Jabal al-'Alā, Jabal al-Ḥaș et Jabal Shbayț) ${ }^{13}$ (figure 6.1), la culture imposait en général au préalable un épierrement soigneux ${ }^{14}$. Cette opération, à l' origine de la formation et de la fossilisation du terroir - quoique, on le verra, certains parcellaires ont subi de profondes modifications en raison de changements de stratégies agricoles -, $s^{\prime}$ inscrit dans la longue durée ${ }^{15}$. L'épierrement n' est en effet qu'une étape d'un processus qui vise à faciliter la culture et, à long terme, avec d'autres techniques, à rendre la terre plus productive.

Les vestiges des épierrements successifs se présentent, d'une part, sous la forme de pierriers et, d'autre part, sous celle d'un parcellaire constitué de murets ou de murs de pierre sèche (figures 6.2 et 6.3).

12 Il faut cependant souligner que certains des parcellaires antiques du Jabal al-'Alā ont pu subir des modifications au cours du temps: d'abord à l'époque ayyoubide, au cours de laquelle une partie des habitations des villages antiques a été réoccupés, ensuite plus récemment, au cours du repeuplement moderne de ces régions $\left(18^{\mathrm{e}}-19^{\mathrm{e}}\right.$ siècles). De nos jours, les remaniements au bulldozer tendent à faire disparaître le parcellaire antique des secteurs basaltiques. Sur la diversité des murs, voir Bernard Geyer, Nazir Awad et Jacques Besançon, "Murs, murets et enclos: l' usage de la pierre dans la mise en valeur des steppes arides de Syrie du Nord," dans De l'île d'Aphrodite au Paradis perdu, itinéraire d'un gentilhomme lyonnais: en hommage à Yves Calvet, éds. Bernard Geyer, Valérie Matoïan et Michel Al-Maqdissi, Ras Shamra-Ougarit XxII (Louvain: Peeters, 2015), 275-298.

13 Ces mesas sont des terres à blé réputées (Abdul-Rahman Hamidé, La région d'Alep: étude de géographie rurale (Paris: Imprimerie de l'Université, 1959), 131, 135). Vraisemblablement en raison de ce potentiel agricole élevé, elles semblent avoir été les premières terres mises en valeur dans l'Antiquité dans les Marges arides de la Syrie du Nord. C'est en effet sur le plateau du Jabal al-'Alā que l'on trouve les inscriptions les plus anciennes de la région (Louis Jalabert, René Mouterde et Claude Mondésert, Inscriptions grecques et latines de la Syrie, vol. 4, Laodicée, Apamène (Paris: Geuthner, 1955), nos. 1871-1872 et 1928). Elles datent au plus tôt du milieu du $2^{\mathrm{e}}$ siècle.

14 Les secteurs basaltiques ne sont vraisemblablement pas les seuls terroirs qui ont été épierrés, mais en raison de leur pierrosité élevée, les vestiges de ces opérations y sont le mieux conservés.

15 Le parcellaire antique des plateaux basaltiques tel qu'il nous apparait aujourd'hui - ou plutôt tel qu'il apparaît sur les photographies aériennes des années 196o - est le produit d'épierrements successifs. 


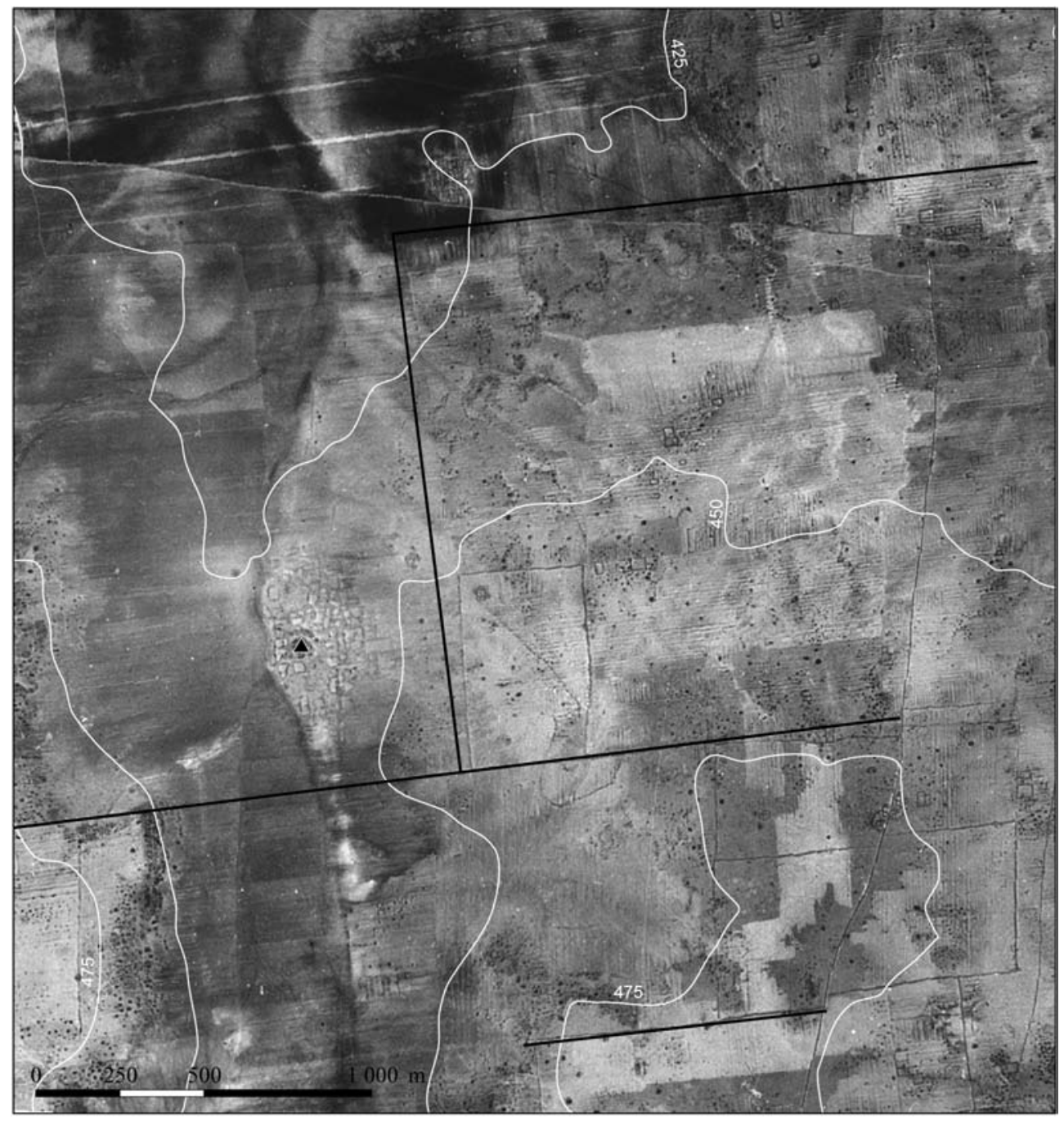

- Site de Samaqiyya al-Šamāliya (probablement byzantin) Photographie aérienne de 1958. — Réseau agraire orthogonal Equidistance des courbes de niveau : $25 \mathrm{~m}$. FIGURE 6.2 Le parcellaire laniéré dans la région de Samaqiyya al-Šamāliyya MARION RIVOAL CMISSION MARGES ARIDES 2011 


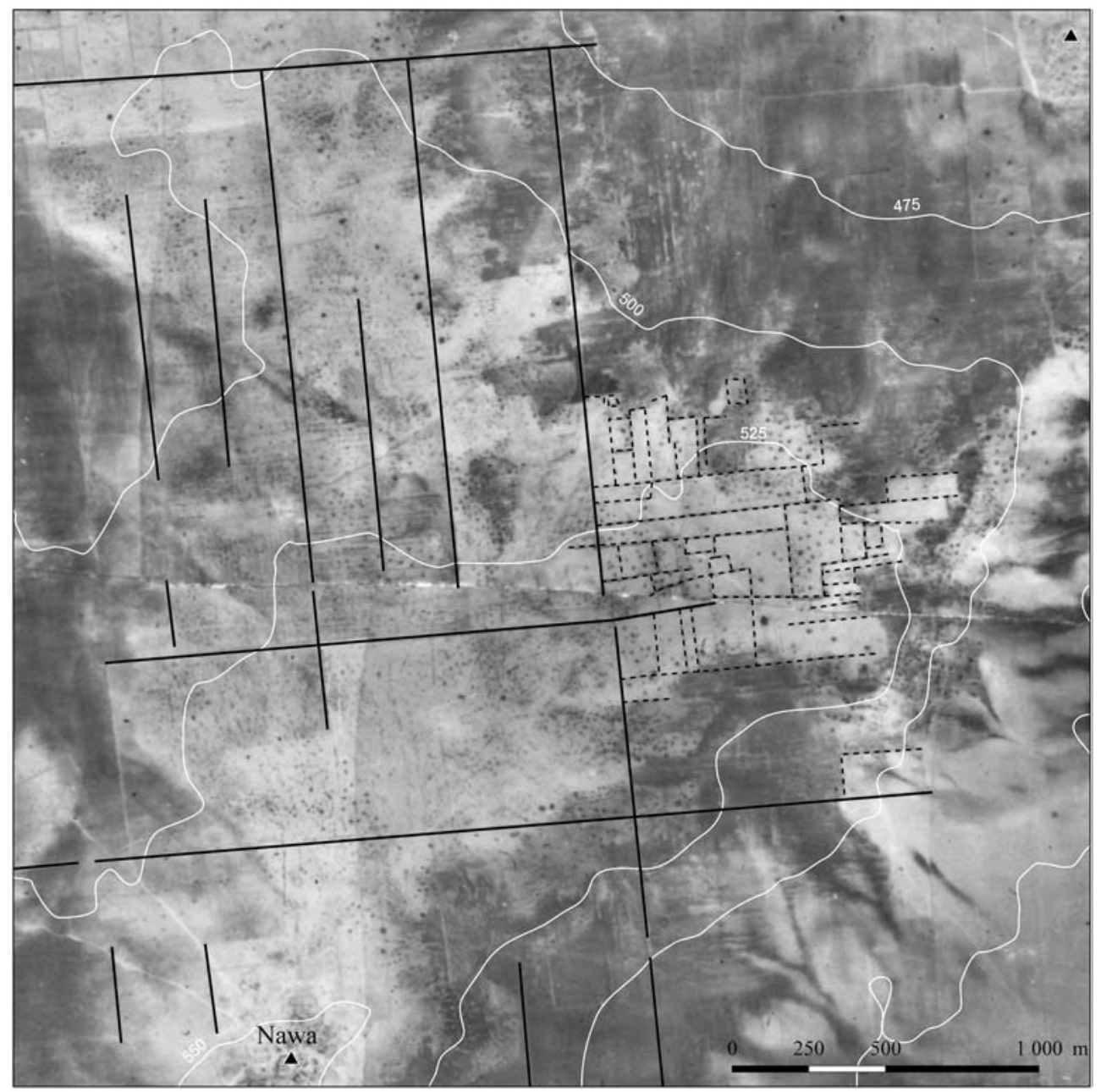

- Site byzantin

Photographie aérienne de 1958.

— Réseau agraire orthogonal

-... Enclos, parcellaire

Equidistance des courbes de niveau : $25 \mathrm{~m}$.

FIGURE 6.3 Réseau agraire orthogonal et parcellaire au nord-ouest de Nawa MARION RIVOAL (CMISSION MARGES ARIDES 2011 
Les pierriers sont, en apparence, de simples empilements de pierres et de blocs ${ }^{16}$, plus ou moins hauts et étendus, souvent approximativement circulaires. Ils sont fréquemment répartis de manière irrégulière à la surface de la parcelle. Leur nombre varie d' un champ à l' autre et il arrive que le produit de l'épierrement d'une même parcelle soit rassemblé en une seule pile fruste de forme allongée, au beau milieu de la surface à cultiver ${ }^{17}$. Les pierriers ne sont pas liés à une forme déterminée du parcellaire, car on les retrouve dans les champs en lanière comme dans les enclos de culture, rectangulaires ou polygonaux, qu' on observe sur le sommet des plateaux et sur leurs versants. La multiplication des pierriers conduit à une réduction parfois importante de la surface cultivable, mais ce constat vaut essentiellement pour les versants des plateaux, dont les sols sont les plus pierreux, et secondairement pour leurs surfaces. Les piémonts et les vallées des mesas ne sont pas exempts de pierres et de blocs de basalte - charriés notamment par les crues des oueds -, ce qui explique que le parcellaire antique soit aussi conservé dans ces zones. En revanche, les pierriers en sont le plus souvent absents, ce qui tendrait à prouver que les exploitants tenaient, sur ces terroirs au potentiel agricole très élevé, à profiter de toute la surface arable. Ce souci s' est notamment traduit par l' aménagement de jardins dans les fonds de vallée ${ }^{18}$. Dans ces secteurs, le produit de l'épierrement a bien servi à la construction des murets qui séparent les différents lopins, mais il est probable que les pierres excédentaires ont été évacuées en dehors des zones de jardins.

\subsection{La trame du parcellaire: les réseaux agraires orthogonaux}

L'épierrement et la mise en valeur du terroir fertile que constitue le Jabal al-'Alā paraissent s'être déroulés dans un cadre organisé. Des alignements réguliers de murets dessinent en effet, sur le sommet de la mesa, une trame orthogonale dans laquelle le parcellaire - en particulier, le parcellaire laniéré - s' insère parfaitement (figures 6.2 et 6.3). Ces réseaux sont particulièrement lisibles, sur les photographies aériennes des années 1960, dans le quart sud-est du plateau ${ }^{19}$. L' imagerie satellitaire, quant à elle, permet d' observer dans le quart nord-ouest

16 Pierre Gentelle a cependant mis en évidence, dans les environs de $\mathrm{Si}$, la structure interne organisée des pierriers de cette partie du Hauran (Pierre Gentelle, "Éléments pour une histoire des paysage et du peuplement du Djebel Hauran septentrional, en Syrie du Sud," dans Hauran I: Recherches archéologiques sur la Syrie du Sud à l'époque hellénistique et romaine, éd. Jean-Marie Dentzer (Paris: Geuthner, 1985), 1:35).

17 Ce type de pierrier peut par exemple être observé sur certains versants du Jabal Shbayṭ.

18 Voir infra.

19 Dans cette zone du Jabal al-'Alā, les alignements couvrent une surface de $18 \mathrm{~km}$ du nord au sud et de $10 \mathrm{~km}$ environ d' ouest en est. 
du plateau ce qui pourrait être le prolongement de la trame identifiée au sud (figure 6.1). ${ }^{20} \mathrm{Si} \mathrm{l}$ 'orientation générale de ces alignements paraît cohérente ${ }^{21}$, seules des études approfondies confrontant l'observation des photographies aériennes et de l' imagerie satellitaire avec des observations et des vérifications de terrain ${ }^{22}$ permettront de définir si ces réseaux orthogonaux relèvent d'une seule et même opération d'organisation du territoire.

À l'heure actuelle, et avec les connaissances qui sont les nôtres, les réseaux agraires suscitent plus d'interrogations sur l' organisation du territoire qu' ils n' apportent de réponses. Leur datation mais aussi leur(s) rôle(s) éventuel(s) dans l'organisation et le contrôle de ce territoire posent question. Cette trame orthogonale traduit-elle une opération d'assignation des terres ordonnée par l'administration impériale? Si oui, précède-t-elle l'occupation du plateau ou reconnaît-elle de fait un partage du territoire déjà effectif entre les différents sites (habitat isolé et/ou groupé?) installés sur le sommet du plateau? S' il s' agit d'une assignation de terres, conserve-t-elle une quelconque valeur fiscale aux $5^{\mathrm{e}}$ et $6^{\mathrm{e}}$ siècles, au moment où les Marges arides de Syrie du Nord connaissent leur principal essor économique et démographique ${ }^{23}$ ? Il est bien difficile de saisir la manière dont s' organise la distribution des sites byzantins (habitat et finage) dans ce cadre. Sur les photographies aériennes des années 196o en effet, les vestiges des murets de délimitation s' estompent aux abords des agglomérations antiques.

20 On peut suivre ces alignements, à cheval sur la partie nord du plateau et le piémont du chaînon calcaire situé à l' ouest (Jabal Zawiya), sur une vingtaine de kilomètres du nord au sud et $12 \mathrm{~km}$ environ d' ouest en est.

21 L'orientation générale est de 6 à $7^{\circ}$ ouest pour les trames identifiées au nord et au sud du plateau. L'intervalle entre les axes nord-sud, dans le quart sud-est du plateau, est en moyenne de 240 à $280 \mathrm{~m}$ et celui entre les axes est-ouest, de 570 à $620 \mathrm{~m}$ environ. Dans le quart nord-ouest, la distance entre les premiers est de 400 à $450 \mathrm{~m}$ et de 430 à $470 \mathrm{~m}$ approximativement entre les seconds. Aucun espace de circulation (limites) n'a encore été reconnu, mais cela ne signifie pas qu' il n' en existait pas. La définition des photographies aériennes anciennes et l'état de conservation actuel de ces alignements ne permettent pas d'approfondir cette question. Dans ce contexte, il est bien difficile de déterminer la nature ou les fonctions de ces réseaux orthogonaux.

22 Les photographies aériennes et l'imagerie satellitaire permettent un relevé relativement précis de ces alignements, mais cette documentation engendre des déformations et des distorsions que seules les observations de terrain sont en mesure de corriger.

23 Rivoal, "Le peuplement byzantin des massifs basaltiques," 233-236. Bernard Geyer et Marie-Odile Rousset, "Les steppes arides de la Syrie du Nord à l'époque byzantine ou la «ruée vers l' est»," dans Conquête de la steppe et appropriation des terres sur les marges du croissant fertile, éd. Bernard Geyer (Lyon: Maison de l'Orient et de la Méditerranée, 2001), $115^{-118 .}$ 
Dans le quart nord-ouest du Jabal al-'Alā, la présence du village byzantin de Hawā et son association avec un enclos de territoire (figure 6.1) ${ }^{24}$ pourraient indiquer l' existence de deux types d'organisation du territoire sur ce plateau. $\mathrm{Au}$ réseau agraire orthogonal identifié au nord-ouest et au sud-est succèderait, sur la frange orientale du plateau, au nord, une forme plus spontanée d'appropriation des terres: l'enclos de territoire. Malheureusement, l' absence d' alignements orthogonaux conservés dans le quart nord-est du Jabal al-'Alā ne permet pas d' étudier les relations chronologiques qu' entretiennent dans cette zone ces deux types d' aménagements. Les enclos de territoire sont cependant probablement postérieurs aux réseaux orthogonaux. Si les alignements qu' on observe sur une grande partie de la surface du plateau correspondent à une forme d' assignation des terres et si les enclos de territoire - comme on le verra relèvent bien d'une forme d'appropriation du terroir plutôt représentative de la période byzantine, on peut douter que la trame orthogonale du Jabal al-'Alā ait conservé aux $5^{\mathrm{e}}$ et $6^{\mathrm{e}}$ siècles les rôles administratifs qui pouvaient être les siens à l'origine. Mais l'absence d'enclos de territoire repéré à ce jour sur le Jabal al-'Alā, exception faite de celui de Hawā, pourrait laisser penser que, sur le reste du plateau, les limites des finages villageois restaient calquées sur les réseaux agraires orthogonaux.

\subsection{Les formes du parcellaire}

Le parcellaire traduit la volonté d'organiser l'espace agraire, non pas à l'échelle régionale, comme on peut le supposer pour les réseaux orthogonaux, mais à celle d'une agglomération. Le parcellaire définit, au sein d'un territoire communautaire, les surfaces cultivables par chaque exploitant et façonne le finage en fonction des cultures pratiquées.

Les plateaux basaltiques de la région portent les vestiges d'un parcellaire diversifié, qui a manifestement été conçu pour accueillir des cultures différentes. Ces aménagements reflètent des orientations économiques complexes, dont certaines paraissent avoir évolué au cours du temps - bien qu'il soit impossible de dater précisément ces modifications.

Plusieurs types de parcelles et d' enclos peuvent être reconnus sur et autour des trois plateaux basaltiques: des champs en lanière, des terrasses de culture, des jardins et, enfin, des enclos de forme et de destination variable en fonction de leur localisation.

24 Rivoal, "Le peuplement byzantin des massifs basaltiques," 225, fig. 10. Ces enclos sont particulièrement fréquents dans le Jabal al-Ḥaș, le Jabal Shbayț et dans la steppe, à l' est du Jabal al-'Alā. Le village de Hawā est l' exemple le plus occidental que nous connaissions. Sur la notion d' enclos de territoire, voir infra. 


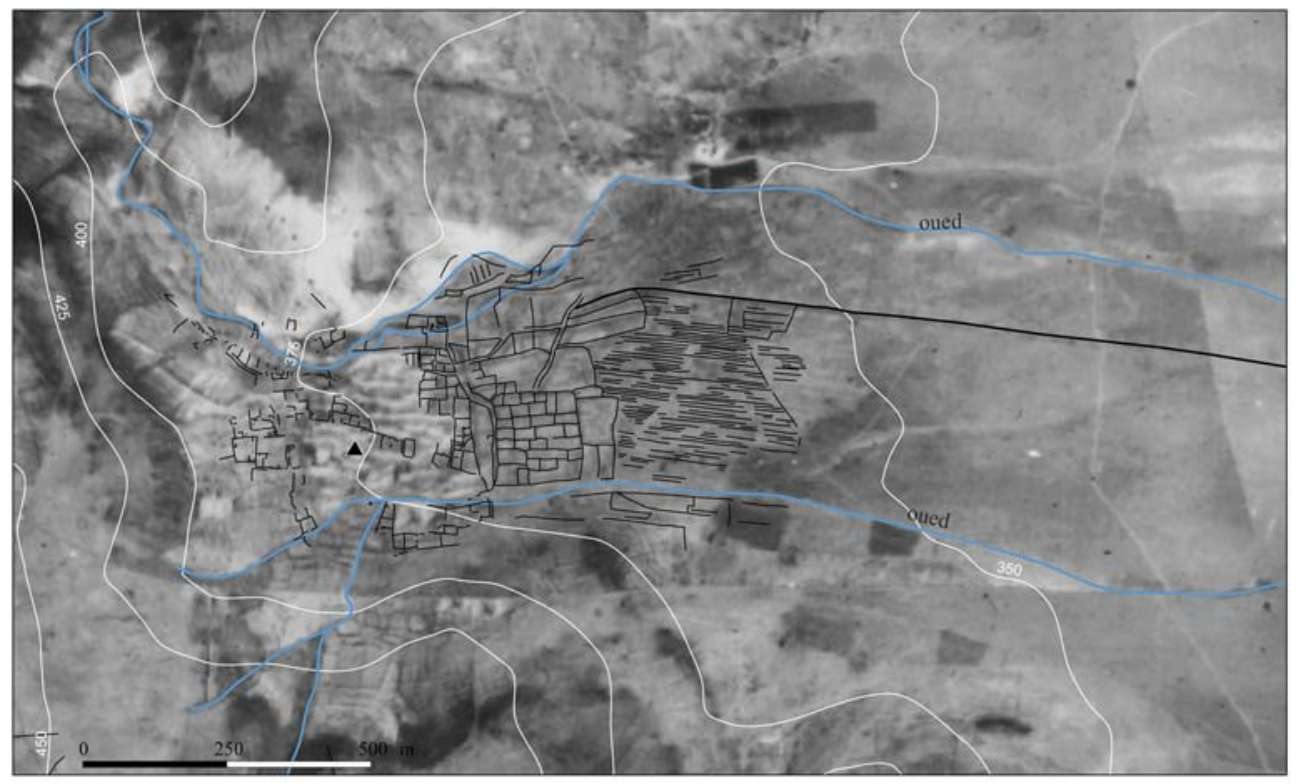

- Site byzantin de Rasm al-Hiajal

Photographie aérienne de 1958.

- Enclos de territoire

_ Enclos de culture, parcellaire laniéré, jardins

Equidistance des courbes de niveau : $25 \mathrm{~m}$.

FIGURE 6.4 Mise en valeur autour du village de Rasm al-Hajal

MARION RIVOAL (C)MISSION MARGES ARIDES 2011

Les champs en lanière sont la forme la plus courante du parcellaire. On les observe sur chacun des sommets des plateaux mais aussi dans certaines vallées du Jabal al-'Alā et sur les bas de versant, dans le sens de la pente, et au débouché de certaines vallées du Jabal Shbayṭ sur le piémont, comme à l' est de Rasm alHajal (figure 6.4).

Ces parcelles très allongées ${ }^{25}$, comparables à celles que l' on connaissait dans l'économie traditionnelle syrienne ${ }^{26}$ avant les opérations de remembrement

$25340 \times 40 \mathrm{~m}$ pour des parcelles antiques d'orientation approximativement est-ouest au nord d'al-Ṭūbā, dans la moitié sud du Jabal al-'Alā ou encore $600 \times 20 \mathrm{~m}$ pour des parcelles d'orientation méridienne dans le même secteur. Sur le sommet du Jabal al-Haș, on observe même par endroits, sur les images satellitaires Google Earth, des champs de moins d'une dizaine de mètres de large pour une longueur de plusieurs centaines de mètres.

26 André Latron évoque notamment le cas du terroir du village moderne de Sfïrā, dont certaines parcelles mesuraient $3500 \mathrm{~m}$ de long pour quelques sillons de large seulement (André Latron, La vie rurale en Syrie et au Liban: étude d'économie sociale (Beyrouth: Imprimerie catholique, 1936), 183 et pl. 2). 
des années 1930 et adaptées aux labours pratiqués à l' araire, sont destinées à la culture d' annuelles et plus spécialement à celle des céréales de plein champ ${ }^{27}$.

Se substituant aux champs en lanière sur les versants les plus raides - c'està-dire ceux soumis au ravinement, soit la quasi-totalité des pentes des plateaux basaltiques du nord ${ }^{28}$-, des terrasses de culture semblent refléter le second pan d' une agriculture principalement bipolaire, du moins dans ce secteur. Ce type d' aménagement renvoie en effet à une pratique intensive de l' arboriculture ${ }^{29}$. Dans le cas qui nous occupe, les éléments de pressoirs qu'ont livrés les prospections du Jabal al-Ḥaș et du Jabal Shbayț ${ }^{30}$ attestent la culture de l'olivier et l'on peut supposer que celle de la vigne s'y ajoutait.

À proximité de certains villages, une autre forme de mise en valeur a été repérée: des jardins ${ }^{31}$. Le potentiel agricole élevé des fonds de vallée, leur étendue restreinte, les possibilités d'irrigation qu' offraient la proximité des oueds et la présence d'une nappe de sous-écoulement, alimentée une bonne partie de l' année sinon toute l' année, ont conduit les villageois à délimiter soigneusement des surfaces de culture beaucoup plus modestes que les autres. Localisées au plus près des villages, ces parcelles présentent une forme régulière, carrée ou rectangulaire, de 20 à $40 \mathrm{~m}$ de long à 20 à $30 \mathrm{~m}$ de large. Il est probable qu' elles recevaient des cultures particulièrement exigeantes en soin et en eau,

27 Jacques Weulersse, Paysans de Syrie et du Proche-Orient (Paris: Gallimard, 1946), 99. Si cette forme de parcelle exclut l'arboriculture, pour des raisons pratiques, la culture de la vigne, elle, reste possible (Latron, La vie rurale, 195).

28 Seule la retombée du plateau du Jabal al-'Alā vers les collines de l' est est souvent assez progressive pour rendre superflue la construction de terrasses.

29 Le complantage n' est pas exclu, mais l'effort considérable que requiert la construction de terrasses de culture ne se justifie guère, dans ce milieu, dans le cadre d' une céréaliculture (Philippe Blanchemanche, Bâtisseurs de paysages : terrassement, épierrement et petite hydraulique agricole en Europe, XVII ${ }^{e}-X I X^{e}$ siècles (Paris: Maison des Sciences de l'Homme, 1990), 135-137).

30 L'architecture en brique crue, caractéristique de ces deux plateaux - l'utilisation du basalte dans la construction est plus importante dans le Jabal al-'Alā, même si la brique crue est loin d'en être absente -, ne permet pas de reconnaitre par la prospection de surface la nature des édifices en ruines, contrairement au Massif calcaire par exemple. Seuls des éléments de pressoir ont été découverts. Les contours des bâtiments qui les abritaient restent difficiles à cerner. Des jumelles, des contrepoids, une table de pressurage et, plus nombreuses, des meules dormantes et des meules roulantes ont ainsi été retrouvés sur une poignée de sites de ces plateaux. Les broyeurs à meule prouvent une production d'huile d' olive. Et, à en juger par la surface qu' occupent les terrasses sur ces terroirs, il est certain que le nombre de pressoirs identifiés est très en-deçà de leur effectif réel.

31 Par exemple Rasm al-Ḥajal et al-Mūallaq, respectivement installés dans une vallée du Jabal Shbayṭ et du Jabal al-Ḥaș, mais aussi Hawā (Jabal al-Alā) et al-Qașțal, un village établi à proximité d'un oued important qui entaille le glacis de piémont des Palmyrénides, à 5 o km environ à l' est de la pointe sud du Jabal al-'Alā. 
et déterminantes pour l'économie domestique. Les superficies des différents lotissements, sensiblement équivalentes, suggèrent - au moins pour l' exemple de Rasm al-Hajal et il est probable qu'il ne s'agit pas d'un cas isolé - une division égalitaire de ce terroir privilégié. Chaque famille jouissait vraisemblablement au minimum d'un de ces lopins ${ }^{32}$.

\subsection{Les enclos}

Aux côtés de ce parcellaire aux formes et aux fonctions relativement bien définies, un grand nombre d'enclos ont également été identifiés. Leur forme, leur surface et leur(s) destination(s) varient considérablement. Les exemples de partition du territoire dont nous avons jusqu' ici signalé l' existence se limitent, pour la plupart, aux plateaux basaltiques. Les enclos, eux, se trouvent dans des contextes beaucoup plus variés et, même s'ils sont représentés sur et autour des mesas, ils sont plus fréquents à l'est et au sud de ces plateaux ${ }^{33}$. Le plus souvent, en l'absence de toute partition interne, c'est à partir de facteurs hydrologiques (présence/absence d' oued) et édaphiques (nature, épaisseur et capacité de rétention en eau du sol, présence/absence d'une dalle calcaire à l'affleurement) ${ }^{34}$ que l'on peut déduire la fonction (culture ou élevage) de certains de ces enclos.

Il existe également une catégorie d' enclos que l' on retrouve sur les plateaux basaltiques et dans la steppe indifféremment et dont la fonction a vraisemblablement plus à voir avec la volonté de délimiter, de revendiquer - et de protéger - une surface agricole. Ce sont ce que nous avons appelé des «enclos de territoire $»^{35}$. Avec les réseaux agraires orthogonaux, ils font partie des vestiges les

32 Le rôle des jardins dans l'équilibre alimentaire familial est notoirement reconnu, que ce soit dans le cadre de l'économie traditionnelle syrienne des années 1930-1940 (Thierry Boissière, Le jardinier et le citadin: ethnologie d'un espace agricole urbain dans la vallée de l'Oronte en Syrie (Damas: Institut Français du Proche-Orient, 2005), 65) ou de l' économie antique en général (Michel Kaplan, "L'économie paysanne dans l'Empire byzantin du ve au x $\mathrm{x}^{\mathrm{e}}$ siècle," dans Byzance: villes et campagnes, éd. Michel Kaplan (Paris: Picard, 2006), 43).

33 Sur les enclos des plateaux basaltiques, voir Rivoal, "Le peuplement byzantin des massifs basaltiques," 227-233. Pour les enclos identifiés dans la steppe, entre Shaykh Hlāl et Ithriyā, voir Rivoal, "Entre steppe et plateaux basaltiques," 152-155.

34 Sur la présence de ces croûtes et leur impact sur la mise en valeur de la région, voir Besançon et Geyer, "Contraintes écogéographiques," 35.

35 La notion d' enclos de territoire a été évoquée par Bernard Geyer, "Des fermes byzantines aux palais omayyades ou l' ingénieuse mise en valeur des plaines steppiques de Chalcidique (Syrie)," dans Aux origines de l'archéologie aérienne: A. Poidebard (1878-1955), éds. Lévon Nordiguian et Jean-François Salles (Beyrouth: Université Saint-Joseph, 2000), 109122, par Besançon et Geyer, “Contraintes écogéographiques," 50-51, par Geyer, Besançon 
plus susceptibles d'éclairer les modalités d'appropriation et d'administration des campagnes des Marges arides.

Ces enclos de territoire se présentent sous la forme de murets de pierre sèche ${ }^{36}$, à double parement, au tracé continu et irrégulier. Ils délimitent une surface variable ${ }^{37}$ au potentiel agronomique souvent très inégal - contrairement aux enclos de culture et d'élevage. Au centre ou en périphérie de ces enclos se trouve toujours - sauf exceptions, sur lesquelles nous reviendrons plus loin - une unique implantation (village, hameau ou encore ferme ou monastère $\left.{ }^{38}\right)$. C' est naturellement le caractère isolé du site d'habitat qui nous amène à y voir une forme de revendication et de possession du territoire défini par l'enclos.

Avec l'économie mixte qui caractérise les Marges arides de la Syrie du Nord ${ }^{39}$ et la place importante qu'occupe l'élevage de caprinés dans le sud et l'est de la région ${ }^{40}$ - zone dans laquelle on a reconnu le plus grand nombre d'enclos de territoire -, il est cependant peu probable que ces enclos circonscrivent l'intégralité du terroir effectivement exploité par un site. Ils définissent presque certainement le territoire exclusif que s' est arrogé un site, un territoire qui rassemble des ressources culturales et pastorales et, le plus souvent, une portion du lit d'un oued. Mais, même dans les secteurs où les enclos de territoire sont particulièrement nombreux, leur maillage est assez lâche pour que les troupeaux d'une ferme ou d'un hameau - mais aussi ceux des pasteurs nomades

et Rousset, "Les peuplements anciens," 61, par Geyer et Rousset, "Les steppes arides," 118 et par Rivoal, "Entre steppe et plateaux basaltiques," 153, n. 47.

36 Il pourrait s' agir, comme pour l'habitat, de soubassements de pierre destinés à recevoir une superstructure en brique mais, dans ce cas, celle-ci n' a guère laissé de trace.

37 De 23 ha pour l' enclos de territoire de la ferme de 'Umm Miyāl 2 (Jabal Shbayt) à 1115 ha pour celui du village de 'Umm Zahmak, dans les collines à l' est du Jabal al-'Alā.

38 Plusieurs plans d' agglomérations associées à des enclos dans Catherine Duvette, "Habitat byzantin dans la steppe: maisons et villages de terre," dans Entre nomades et sédentaires. Prospections en Syrie du Nord et en Jordanie du Sud, éds. Pierre-Louis Gatier, Bernard Geyer et Marie-Odile Rousset (Lyon: Maison de l'Orient et de la Méditerranée, 2010), 177-191 et Catherine Duvette, "Rasm Afandi, village des steppes de Syrie," dans Habitat et environnement: prospections dans les Marges arides de la Syrie du Nord, éds. Marie-Odile Rousset, Bernard Geyer, Pierre-Louis Gatier et Nazir Awad, Travaux de la Maison de l'Orient 71, Conquête de la steppe IV (Lyon: Maison de l'Orient et de la Méditerranée, 2016), 153-168.

39 Voir Geyer et Rousset, "Les steppes arides," 115-118; Geyer, Besançon et Rousset, "Les peuplements anciens," 61-69; Rivoal, "Le peuplement byzantin des massifs basaltiques," 227233 et Rivoal, "Entre steppe et plateaux basaltiques," 161.

40 Geyer, "Des fermes byzantines," 116-117; Marie-Odile Rousset et Catherine Duvette, "L'élevage dans la steppe à l'époque byzantine: indices archéologiques," dans Les villages dans l'Empire byzantin, $I V^{e}-X V^{e}$ siècle, éds. Jean Lefort, Cécile Morrisson et Jean-Pierre Sodini (Paris: Lethielleux, 2005), 485-494; Rivoal, "Entre steppe et plateaux basaltiques," 16o-161. 
dont la présence, au moins saisonnière, est probable ${ }^{41}$ - aient pu paitre hors leurs limites, sans pour autant empiéter sur le territoire du site voisin. On doit aussi garder à l' esprit qu'il y a de grandes chances pour que, les années où les précipitations étaient les plus abondantes, les exploitants d' un village aient mis en culture les fonds d' oueds et les dépressions les plus propices, à l' extérieur des limites des enclos de territoire, dans ces zones qui paraissent non attribuées - ou non revendiquées.

Les enclos de territoire identifiés à ce jour - sur le terrain ou à partir des photographies aériennes anciennes et de l'imagerie satellitaire - restent peu nombreux relativement au nombre de sites antiques et islamiques reconnus et la restitution de leur tracé demeure fréquemment incomplète. Mais il nous semble que le rôle de ces aménagements, ainsi défini, prête peu à controverse. Les enclos de territoire identifiés autour des plateaux basaltiques du nord, du village de Hawā dans le Jabal al-'Alā ou encore dans la steppe au sud d'Andarīn ou aux environs de Shaykh Hlāl paraissent se conformer à la définition que nous proposions plus haut.

En revanche, plus à l' est, autour d'Ithriyā, et sur le bastion montagneux au sud de l'agglomération (Jabal Ithriyā) ${ }^{42}$, des aménagements, pourtant similaires, soulèvent davantage de questions. L' agglomération d' Ithriyā, par exem-

41 Les prospections ont permis d'établir une présence importante des populations d'éleveurs nomades ou semi-nomades dans la zone dite des «Marges arides de Syrie du Nord» de la fin de l'époque hellénistique à l'époque romaine incluse, d'une part, et au cours la période ayyoubide, d'autre part (Marie-Odile Rousset, "Le peuplement de la steppe de Syrie du Nord entre le II ${ }^{\mathrm{e}}$ s. av. et le IV e s. apr. J.-C.," Syria 88 (2011): 123-139). Comme le montrent les citernes (nous le verrons plus loin), les principales aires de nomadisme se trouvent, à l' époque byzantine, à l' est du bastion d' Ithriyā (Geyer et Rousset, "Les steppes arides," 118 et Rivoal, "Entre steppe et plateaux basaltique," 143), mais cela n' exclut pas pour autant la présence occasionnelle - saisonnière? - de pasteurs nomades dans cette partie de la steppe.

Le temple romain étudié par Rudiger Gogräfe témoigne de l'occupation romaine tardive du site (Rudiger Gogräfe, "Der Tempel von Isriye zwischen nahötlicher Kulttradition und römischer Architektur," Topoi 7, no. 2 (1997): 8o1-836). L'occupation byzantine, elle, s' est traduite par la construction de trois à quatre églises et le site figure sur la Notitia Dignitatum (Notitia Dignitatum, éd. Otto Seeck, Notitia dignitatum. Accedunt Notitia urbis Constantinopolitanae et Laterculi provinciarum (Berlin: Berolini, 1876), 33), dont la partie concernant l' Orient est datée du tout début du $5^{\mathrm{e}}$ siècle (Constantin Zuckerman, "Comtes et ducs en Égypte autour de l' an 400 et la date de la Notitia Dignitatum Orientis," Antiquité Tardive 6 (1998): 146). L' identification de Sériane avec Ithriyā est aujourd' hui communément admise (René Dussaud, Topographie historique de la Syrie antique et médiévale (Paris: Geuthner, 1927), 275; Gatier, “« Grande»," 103), malgré les arguments qu'Ernst Honigmann avançait en faveur d' al-Sa'an (Ernst Honigmann, "Syria," dans Paulys Realencyclopädie der Classischen Alterumswissenschaft 4A, 2, éds. Georg Wissowa, Karl Mittelhaus et Wilhelm Kroll (Stuttgart: J.B. Metzler, 1932), col. 1677). 
ple, est ceinte d' un vaste enclos - sous réserve qu'il lui soit contemporain - qui délimite une superficie d' environ $75 \mathrm{~km}^{243}$. Mais, contrairement à ce que l' on observe dans le cas des enclos de territoire, cette surface n' est pas tout à fait dépourvue de sites antiques (figure 6.5).

On y trouve en effet de petites implantations, occupées pendant la période byzantine et au-delà: un monastère connu par une inscription (al-Ṭāhūna 1) ${ }^{44}$ et un second site, perché comme le premier, qui pourrait également être de même nature (Khunșur Ithriyā al-Shamālī) ${ }^{45}$. Aucune agglomération ne s'est donc développée dans l' espace circonscrit par cet enclos et la seule présence, à l'intérieur de ses limites, de ces deux sites d'habitat isolé ne suffit pas à écarter définitivement la fonction d'enclos de territoire. Car les liens de dépendance administrative entre les sites sont loin d'avoir été élucidés dans la région et il est tout à fait envisageable que les deux sites soient rattachés fiscalement à l'agglomération d'Ithriyā. L'enclos qui entoure la base du promontoire sur lequel est établi le monastère d'al-Ṭāhūna 1 pourrait indiquer une enclave et une forme de concession, au sein du vaste territoire d'Ithriyā ${ }^{46}$.

Plus au sud, sur le bastion d'Ithriyā, des aménagements présentent aussi des points communs avec les enclos de territoire reconnus au nord et à l' ouest (figure 6.5). Agglutinés les uns aux autres selon un axe nord-sud, ils semblent

43 L'enclos de territoire d' Ithriyā n' avait jusque là été que partiellement publié dans Geyer, "Des fermes byzantines," 120 et Geyer, "Murs, murets et enclos," 292. Nous remercions Bernard Geyer de nous avoir autorisées à publier cet ensemble.

44 Mouterde et Poidebard, Le Limes de Chalcis, 1:91, 226. Sur ce site a été retrouvée de la céramique byzantine et abbasside, en faible quantité, la majorité des tessons identifiés relevant de l'époque omeyyade.

45 Ce site a connu une occupation byzantine, omeyyade et abbasside - toutefois, ces deux dernières périodes sont moins bien représentées par la céramique. Il a livré également une monnaie de Maurice Tibère (frappée en 589-59o) et un fragment de dirham d' argent abbasside: Callot, "Monnaies," nos. 42 et 64 . Ce site pourrait aussi avoir eu une fonction plutôt résidentielle: Bernard Geyer et Marie-Odile Rousset, "Déterminants géoarchéologiques du peuplement rural dans les Marges arides de Syrie du Nord aux VII ${ }^{\mathrm{e}}$-IX $\mathrm{X}^{\mathrm{e}}$ siècles," dans Le Proche-Orient de Justinien aux Abbassides, Peuplement et dynamiques spatiales, éds. Antoine Borrut et al. (Turnhout: Brepols, 2011), 88. Voir aussi Denis Genequand et Marie-Odile Rousset, "Résidences aristocratiques byzantines et omeyyades des marges arides du nord de la Syrie," dans Habitat et environnement: prospections dans les Marges arides de la Syrie du Nord, éds. Marie-Odile Rousset, Bernard Geyer, Pierre-Louis Gatier et Nazir Awad, Travaux de la Maison de l' Orient 71, Conquête de la steppe IV (Lyon: Maison de l'Orient et de la Méditerranée, 2016), 220-225.

46 François Villeneuve supposait que les villages de Syrie du Sud avaient accordé à des monastères, plus tardifs, des concessions sur leur finage (François Villeneuve, "L'économie rurale et la vie des campagnes dans le Hauran antique ( $\mathrm{I}^{\mathrm{er}}$ s. av. J.-C.-VII $\mathrm{I}^{\mathrm{e}}$ s. ap. J.-C.): une approche," dans Hauran I: Recherches archéologiques sur la Syrie du Sud à l'époque hellénistique et romaine, éd. Jean-Marie Dentzer (Paris: Geuthner, 1985), 1:121). 


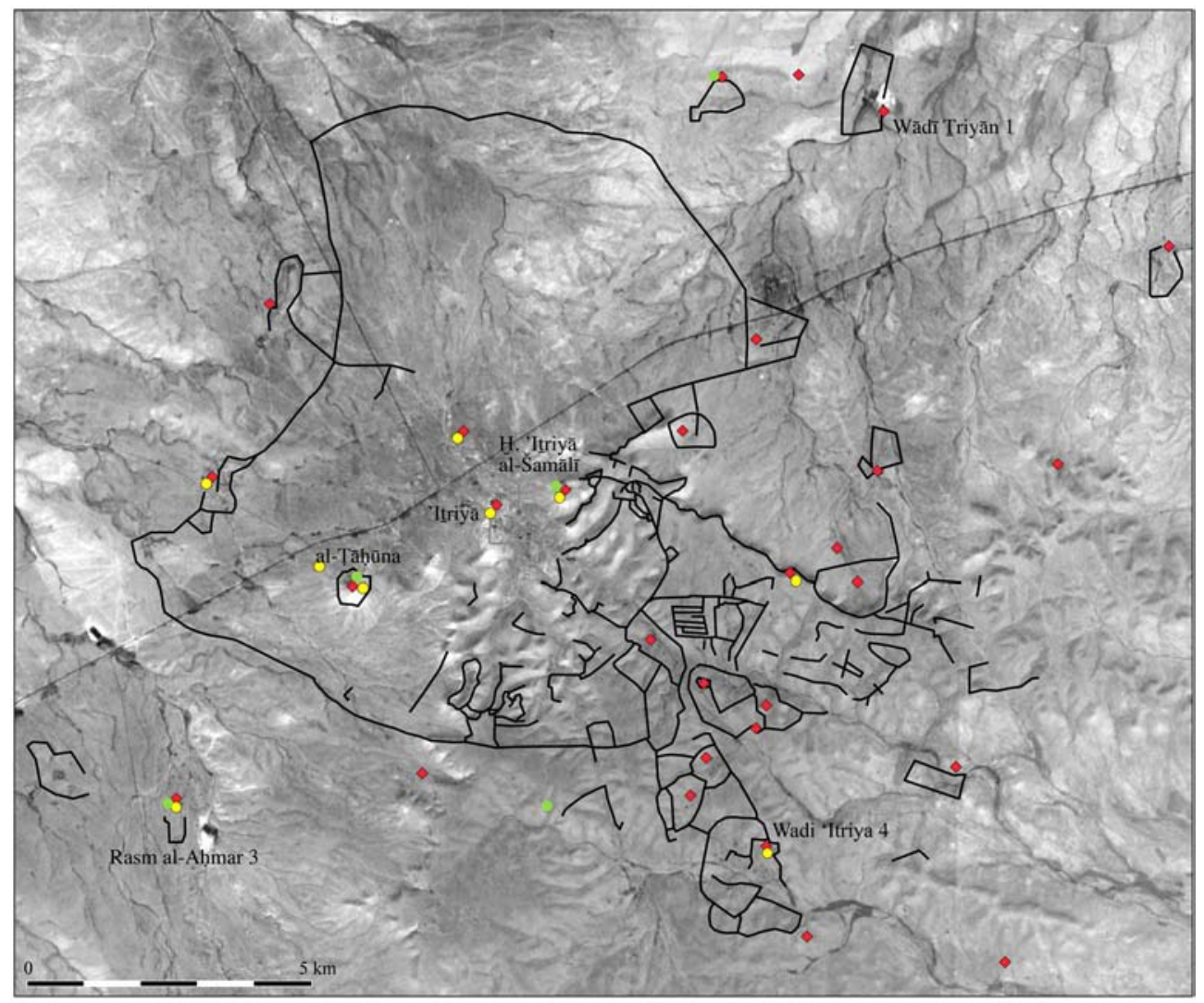

- Site occupé à la période byzantine

FIGURE 6.5 Le grand enclos de 'Ithriyā

BERNARD GEYER, MARION RIVOAL, MARIE-ODILE ROUSSET CMISSION

MARGES ARIDES 2018

longés à l' est par un axe de circulation qui devait traverser les Palmyrénides en direction du sud (figure 6.1). Certains de ces enclos satisfont tous les critères des enclos de territoire, c' est-à-dire qu' ils sont associés à un site archéologique et marquent les limites de son terroir potentiel, en incluant le plus souvent un fond de vallée ${ }^{47}$. Mais d' autres enclos, par exemple situés au sud du monastère de Wādī Ithriyā 4, paraissent n' offrir aucune alternative à l'élevage. Bien qu' ils

47 Les fonds de vallée ne sont guère entaillés sur ce bastion montagneux, les possibilités de culture y sont très réduites. 
jouxtent d'autres enclos, ils ne semblent curieusement rattachés à aucun site. Leur rôle est donc manifestement distinct de celui des enclos de territoire. Mais lorsque l'on connaît l'orientation économique spécifique de ce secteur, tourné vers l'élevage ${ }^{48}$ (élevage de caprinés certainement, mais aussi possiblement de chevaux pendant la période de cantonnement de la garnison d'equites scutarii Illyriciani, sous Dioclétien $)^{49}$, ces enclos, comme une grande partie de celui qui entoure Ithriyā, pourraient correspondre à des aires de pâture réservées et renvoyer à une gestion raisonnée, alternée, de la ressource pastorale. Le potentiel agronomique médiocre de ces surfaces encloses ne permet guère d' envisager d'une autre forme de mise en valeur ${ }^{50}$.

\section{De rares indications sur la datation des structures agraires et les différentes politiques de mise en valeur de la région}

L'étude des structures agraires n'éclaire qu'indirectement les modalités d'appropriation du terroir et l'organisation et la gestion des campagnes. Les ouvrages hydrauliques, on le verra, sont susceptibles d'apporter des réponses partielles à ces questions, mais on peut aussi exploiter dans ce but d'autres types de données - bien qu' elles soient plus hétérogènes et plus diffuses. Le réseau routier - encore qu' il soit difficile de déterminer la durée d'utilisation des voies romaines et des routes secondaires - et de rares inscriptions peuvent nous aider à préciser la situation.

L'impression qui se dégage du réseau routier des Marges arides de la Syrie du Nord - que l' on peut tenter de reconstituer à partir des documents cartographiques antiques, des sources historiques et des données archéologiques ${ }^{51}$ est celle d'une sous-administration de la région, un trait qu' avait déjà souligné Pierre-Louis Gatier, en constatant le développement limité et tardif des cités de

48 Geyer, "Des fermes byzantines," 116-118; Rivoal, "Entre steppe et plateaux basaltiques," 160-161.

49 Voir Rivoal, "Entre steppe et plateaux basaltiques," 162.

50 Le bastion d'Ithriyā présente en effet une surface largement décapée par l' érosion (Besançon et Geyer, "Contraintes écogéographiques," 17-22).

$5^{1} \quad$ Nous ne reprendrons pas ici les résultats, bien connus, auxquels sont parvenus André Poidebard et René Mouterde à partir d'observations aériennes (Mouterde et Poidebard, Le Limes de Chalcis). D' autres ont mis en cause, non pas tant leurs méthodes de travail, que leur fâcheuse tendance à amalgamer des réseaux d'époques différentes (Pierre-Louis Gatier, "Une frontière sans limes?" dans Aux origines de l'archéologie aérienne: A. Poidebard (1878-1955), éds. Lévon Nordiguian et Jean-François Salles (Beyrouth: Université Saint-Joseph, 2000), 142). 
la région ${ }^{52}$. Les terres agricoles parmi les plus fécondes, les plateaux basaltiques en particulier, apparaissent en effet relativement à l' écart des principaux axes de communication, au moins jusqu' au $7^{\mathrm{e}}$ siècle (figure 6.1). La situation évolue au début de l'époque islamique, à partir du moment où les voyageurs qui se rendaient d'Alep à Bagdad ont préféré emprunter la route du désert pour bénéficier de la protection des Bédouins ${ }^{53}$. L'axe Khanāṣir - Sūriyā (Ithriyā) par al-Ḥammām et al-Zarqa' devient alors prépondérant, jusqu' au milieu du $10^{\mathrm{e}}$ siècle $^{54}$.

Les inscriptions du Jabal al-'Alā, quant à elles, prouvent, d'une part, une occupation relativement précoce de la partie sud du plateau ${ }^{55}$ et, d' autre part, la mainmise tout aussi précoce de l'administration impériale sur ces terres. L'une de ces inscriptions, retrouvée à proximité d'un village byzantin à Shaykh 'Alī Qāsūn, au sud du Jabal al-Alā, fait référence à la borne d'un praetorium, mise en place en $296 / 297$ ap. J.-C., sous la Tétrarchie ${ }^{56}$. Quel que soit le sens à donner à l'emploi du terme praetorium - domaine impérial, comme le suggère Marc Griesheimer ou, plus vraisemblablement, relais en contexte routier, comparable à une taberna ${ }^{57}$ - cette borne confirme que l' autorité impériale s'exerçait dans les campagnes des Marges arides dès la fin du $3^{\mathrm{e}}$ siècle (mais était-ce encore le cas aux $5^{\mathrm{e}}-7^{\mathrm{e}}$ siècles?). La borne d'Andrôna marque, au $6^{\mathrm{e}}$ siècle, une des limites du territoire de cette ville d' environ 16 o ha, qui n' a jamais été élevée au rang de cité ${ }^{58}$. Le texte de l'inscription suivante, retrouvée dans

52 Gatier, “«Grande»," 106.

53 Ibn Ḥawqal, Kitāb șūrat al-ard, trad. Johannes Hendrik Kramers et Gaston Wiet (Beyrouth/Paris: al-Laǵna ad-duwalìya li-tarǵamat ar-rawāì et G.P. Maisonneuve et Larose, 1964), 176.

54 al-Zarqa' ('Ayn al-Zarqa) est mentionnée en tant que source, à propos d'un épisode de la lutte entre Sayf al-Dawla et les Banu 'Amr au milieu du $1^{\text {e }}$ siècle: al-Bakrī, Kitāb almasālik wa-l-mamālik: juz'ān, éds. Adrien van Leeuwen et André Ferré (Carthage-Tunis: Bayt al-Hakima Dār al-'Arabiyya lil-Kitāb, 1992), 76o. D' après Yāqūt al-Rūmi, Kitāb mu ǧğam al-buldān, éd. Ferdinand Wüstenfeld (Leipzig: F.A. Brockhaus, 1866-1873), 2 :924, l' eau n'y tarit jamais. Sur l' abandon de cette route: Ibn ḥawqal dans Alois Musil, Palmyrena:A Topographical Itinerary (New York: Ams Press, 1928), 119.

55 Voir supra, note 13.

$5^{6}$ Marc Griesheimer, "L' occupation byzantine sur les marges orientales du territoire d' Apamée de Syrie (d' après les inscriptions de Taroutia empôron et d'Androna)," dans Conquête de la steppe et appropriation des terres sur les marges du croissant fertile, éd. Bernard Geyer (Lyon: Maison de l'Orient et de la Méditerranée, 2001), 142-144. À noter que Denis Feissel avait déjà jugé cette hypothèse infondée (Denis Feissel, en collaboration avec PierreLouis Gatier, "Bulletin épigraphique: Syrie, Phénicie, Palestine, Arabie," Revue des Études grecques 116 (2003): no. 565).

57 Corpus Inscriptionum Latinarum, Voluminis tertii: inscriptiones Asiae provinciarum Europae graecarum Illyrici latinae Pars posterior, éd. Theodor Mommsen (Berlin: Berolini, 1873), no. 6123 .

58 Pierre-Louis Gatier, "Une borne d'Andrôna", dans Habitat et environnement: prospections 
le village byzantin de Khirbat Msharfa, mentionne le curateur d'une maison divine ${ }^{59}$ que son rang de magnificus permet de situer dans la seconde moitié du $4^{\mathrm{e}}$ siècle $^{60}$. Elle témoigne donc de l'existence de domaines impériaux dans la partie sud du Jabal al-'Alāel ${ }^{-1}$ Mais, à partir des rares données que fournit l'épigraphie, il est impossible de se faire une opinion précise de l'emprise qu' exerçait l'administration de l'empire sur les campagnes de la région et encore moins de son évolution.

L' interprétation chronologique des vestiges agraires soulève bien des difficultés, car on ne possède d'éléments de datation - et encore, relatifs uniquement - que pour certains d'entre eux. Nous n' avons ainsi aucune idée de la période au cours de laquelle le réseau orthogonal du Jabal al-Alā a été mis en place. Mais on peut remarquer que la plus grande partie du parcellaire laniéré - et, partant, l'exploitation céréalière de ce plateau - se conforme à ce cadre imposé et paraît ne s' en être jamais complètement affranchi - à tout le moins dans les zones où ces alignements sont conservés. Lorsque le paysage ne conserve aucune trace de ce cadre, comme autour du village du Hawā ou encore de celui de Umm Zahmak, à cheval sur les collines basaltiques de l'est du Jabal al-Alā, ce sont les enclos de territoire qui semblent dicter les changements d' orientation du parcellaire, selon que les limites de champs s' appuient à l' intérieur ou à l' extérieur de ces enclos (figure 6.6).

Autour de Drīb al-Wāwī, dans la partie sud du Jabal Shbayț, les enclos de culture (consacrés à l'orge?) ou d'élevage s' adossent également à l' enclos de territoire du village et sont indéniablement postérieurs ${ }^{62}$.

Sur les enclos de territoire eux-mêmes, nous n' avons aucun élément de datation absolue et les indications que la chronologie relative peut apporter sont ténues. Seuls certains des sites associés à ces enclos ont été datés, d'après la céramique collectée en surface. Sur 43 d'entre eux ${ }^{63}, 16$ ont été occupés à la période romaine ou romaine tardive, 36 ont connu une occupation byzantine et 14 une occupation omeyyade. Pour 17 des sites qui ont livré de la céramique

dans les Marges arides de la Syrie du Nord, éds. Marie-Odile Rousset, Bernard Geyer, PierreLouis Gatier et Nazir Awad, Travaux de la Maison de l' Orient 71, Conquête de la steppe IV (Lyon: Maison de l'Orient et de la Méditerranée, 2016), 137-151.

59 Jalabert et Mouterde, Inscriptions, no. 1905.

6o Michel Kaplan, Les hommes et la terre à Byzance du vI ${ }^{e}$ au XI ${ }^{e}$ siècle (Paris: Publications de la Sorbonne, 1992), 141.

61 À noter que le site (un village administré par un komarque) était déjà connu comme un domaine grâce à une inscription datée de 344 (Jalabert et Mouterde, Inscriptions, no. 1908). Le bien-fonds était alors géré par un intendant (pragmateutès).

62 Rivoal, "Le peuplement byzantin des massifs basaltiques," 226, fig. 11.

6322 sites ont connu plusieurs périodes d' occupation. 


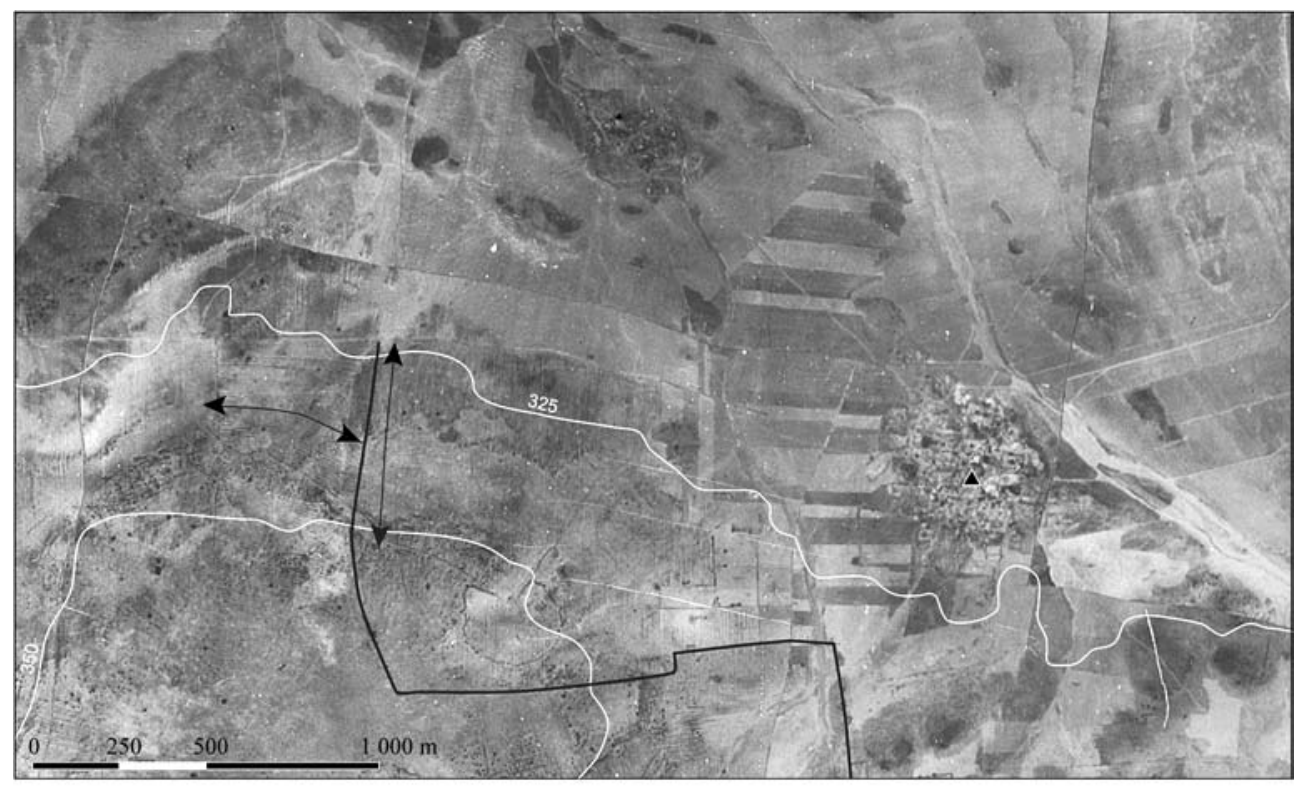

- Site byzantin de 'Umm Zahmak

Photographie aérienne de 1958.

— Enclos de territoire

\section{$\longleftrightarrow$ Orientation du parcellaire laniéré}

Equidistance des courbes de niveau : $25 \mathrm{~m}$.

FIGURE 6.6 Changement de parcellaire à l' ouest de Umm Zahmak

MARION RIVOAL CMISSION MARGES ARIDES 2011

byzantine, l' occupation semble limitée à cette seule période. Par comparaison, seuls quatre sites omeyyades et un site romain n' auraient connu qu' une seule période d'occupation. Enfin, neuf sites omeyyades présentaient des tessons attestant également d'une occupation byzantine. À partir de cet échantillon, certes restreint, on est tenté de conclure que les enclos de territoire sont une forme d'appropriation du territoire plutôt caractéristique de la période byzantine, même si un site romain fait figure de précurseur - pour les 15 autres sites de la même période, l' enclos de territoire pourrait être contemporain ${ }^{64}$ mais il

64 Pour les périodes romaine et romaine tardive, les sites identifiés dans la région semblent principalement tournés vers des activités d'élevage. Ces périodes d'occupation et ces stratégies d'exploitation de la steppe se traduisent souvent sur le terrain par des vestiges d'habitat associés à des cavités aménagées sous roche ou sous la dalle calcaire (Rousset, "Le peuplement de la steppe," 127-129). Le site romain de Rasm M'ammash fait figure d'exception, d'une part, parce qu'il est le seul pour cette période que l'on puisse incontestablement rattacher à un enclos de territoire, et, d'autre part, parce qu'il s' agit d'une ferme, un type d'implantation et une forme de mise en valeur encore peu courants pour cette époque (Marie-Odile Rousset, "Qanats de la steppe syrienne," dans Entre 
pourrait aussi être attribué à la période byzantine - et si une poignée de sites islamiques présentent le même type d'enclos. Mais il est difficile de pousser plus avant la réflexion sur le contrôle et l'organisation des campagnes en se fondant sur les vestiges agraires.

L' antériorité des alignements orthogonaux du Jabal al-'Alā sur le parcellaire laniéré est acquise, tout comme il est évident que les deux types d'aménagement ont fonctionné ensemble et ce probablement sur une longue période. C' est bien ce que l' on peut déduire des alignements de pierriers qu' on observe au nord du village de Nawa (figure 6.3), dans le quart sud-est de ce plateau. Des rangées de pierriers, régulièrement espacées, témoignent d'une modification du parcellaire laniéré initial, vraisemblablement en lien avec un changement d'orientation de la production ${ }^{65}$. Seul l'abandon de la céréaliculture - ou plus probablement une réduction des surfaces consacrées aux céréales - peut expliquer ce remaniement, qui a dû être opéré au profit d' une culture plus rentable, comme l' olivier et, dans une moindre mesure, la vigne - car cette dernière, peut s' accommoder de parcelles en lanière.

Les ouvrages hydrauliques

Entre le $5^{\mathrm{e}}$ et le $10^{\mathrm{e}}$ siècle, le paysage agricole de la région à l' est d'une ligne Salamiyya/Qinnasrin s'est vu profondément modifié par la mise en place de réseaux hydrauliques ${ }^{66}$. Ces éléments du système de mise en valeur généralisé

nomades et sédentaires: prospections en Syrie du Nord et en Jordanie du Sud, éds. PierreLouis Gatier, Bernard Geyer et Marie-Odile Rousset (Lyon: Maison de l'Orient et de la Méditerranée, 2010), 255).

65 Rousset, "Le peuplement de la steppe," 126-127. On ne possède aucune certitude quant au fait que cette réorganisation soit intervenue à la période byzantine, mais, compte-tenu de la forte spécialisation économique de la région à cette époque, cela nous paraît plus que probable. Si les champs en lanière sont, par leur forme même, adaptés au passage de l'araire et donc à la céréaliculture, leur démantèlement implique manifestement une modification des cultures. Dans l'économie traditionnelle syrienne, le parcellaire laniéré est généralement remanié pour faire la place à des champs plus larges, plus propices à l' arboriculture (Latron, La vie rurale, 195-196). Il est probable que les mêmes motivations aient produit les mêmes résultats dans l' Antiquité.

66 La question des qanats dans les Marges arides a été abordée dans plusieurs articles: Bernard Geyer, "Pratiques d' acquisition de l' eau et modalités de peuplement dans les Marges arides de la Syrie du Nord," dans Stratégies d'acquisition de l'eau et société au MoyenOrient depuis l'Antiquité, éds. Mohamed al-Dbiyat et Michel Mouton (Beyrouth: Presses de l'Ifpo, 2009), 25-43; Geyer, Besançon et Rousset, "Les peuplements anciens," 65-68; Geyer et Rousset, "Les steppes arides," 115-117; Rousset, "Qanats de la steppe," 241-270; 
de la région sont, comme les autres, parfaitement adaptés aux particularités géographiques et aux ressources hydriques des différents micro-milieux qui composent les Marges arides de la Syrie du Nord ${ }^{67}$. Les qanats sont des galeries drainantes qui concentrent les eaux des nappes phréatiques superficielles ou des sources et les acheminent par gravité jusqu'à leur débouché, en suivant, souvent sur plusieurs kilomètres, une pente très faible. Des puits d'aération qui servent, au moment de la construction et des opérations de maintenance, à l'évacuation des déblais, sont creusés à intervalles réguliers, entre la galerie souterraine et la surface. Selon la nature des formations géologiques traversées, les parois des puits et de la galerie peuvent être laissées brutes ou maçonnées. Enfin, dans le cas d' un terrain absorbant, des conduits en céramique peuvent être utilisés.

L' irrigation a vraisemblablement permis d' affecter des terres à des cultures exigeantes en eau et contribué à augmenter les rendements, notamment dans la grande fayda centrale. Cette plaine alluviale, vers laquelle converge la plupart des galeries, bénéficie de sols épais et bien drainés, mais, en dehors des années particulièrement pluvieuses, les précipitations y sont trop modiques et les écoulements trop profonds pour profiter aux cultures pluviales. Sa mise en valeur nécessite donc d'irriguer.

Les aménagements hydrauliques repérés lors de la prospection des Marges arides de Syrie du Nord sont de plusieurs types: les galeries drainantes évoquées plus haut et des aménagements greffés sur les sources artésiennes, qanats complétées d'ouvrages divers, donc des systèmes mixtes. On compte environ une quarantaine de ces systèmes dans la zone qui correspond à la carte de Salamiyya au 1: $200000^{e}$, en dehors de la cuvette exoréique de Salamiyya ${ }^{68}$ (figure 6.7).

Jaubert et al., Land Use, 59-6o. Ce réseau est également étudié par la mission britannique d'Andarīn sous la direction de Marlia Mundell Mango (université d' Oxford): Marlia Mundell Mango, "Excavations and Survey at Androna, Syria:The Oxford Team 1999," Dumbarton Oaks Papers 56 (2002): 307-315; Marlia Mundell Mango, "Baths, Reservoirs and Water Use at Androna in Late Antiquity and the Early Islamic Period," dans Residences, Castles, Settlements: Transformation Processes from Late Antiquity to Early Islam in Bilad al-Sham, éds. Karin Bartl et Abd al-Razzaq Moaz (Damas: Deustches Archäologisches Institut, 2008), 73-88.

67 Geyer et Rousset, "Les steppes arides;" Besançon et Geyer, "Contraintes écogéographiques;" Franck Braemer et al., "Conquest of New Lands and Water Systems in the Western Fertile Crescent (Central and Southern Syria)," Water History 2 (2010): 91-114 et fig. 8.

68 Cette zone, au potentiel agricole très riche, a été laissée de côté par la prospection «Marges arides», qui s' est concentrée sur des secteurs plus pertinents pour étudier les rapports hommes/milieu en contexte aride. 


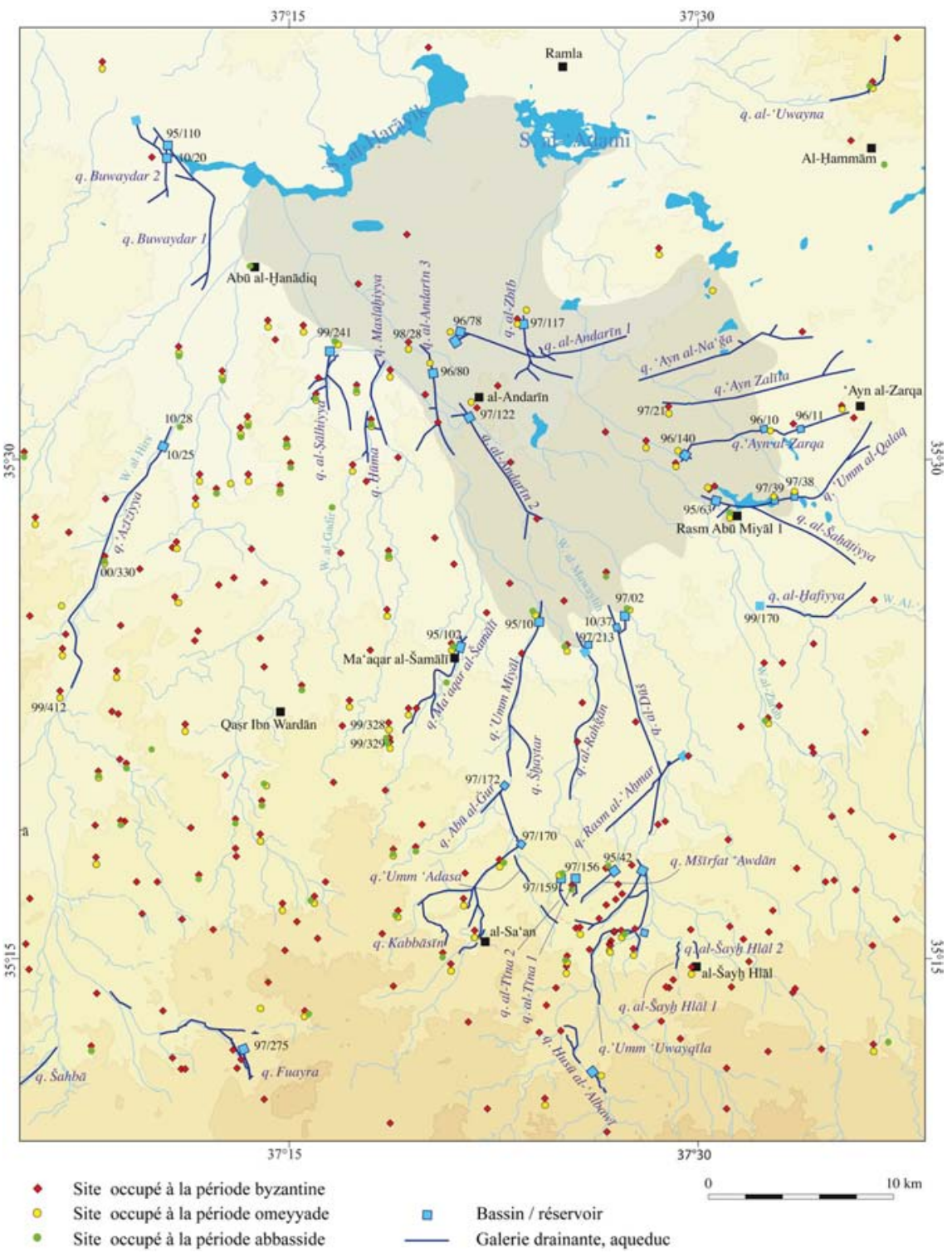

FIGURE 6.7 Carte générale des ouvrages hydrauliques dans les Marges arides et sites prospectés

OLIVIER BARGE, MARIE-ODILE ROUSSET @MISSION MARGES ARIDES 2010 
Les qanats ont été cartographiées d'après les informations fournies par les cartes topographiques au 1: 50 oooe $\mathrm{d}^{\mathrm{d}}$ après des photographies aériennes anciennes (vues verticales à l'échelle approximative de $1: 80000^{\mathrm{e}}$, prises en 1958 et 1961) et des vues d'hélicoptère ${ }^{69}$, des images Landsat et Google Earth mais aussi, et surtout, grâce aux informations collectées sur le terrain. La prospection «Marges arides», réalisée entre 1995 et 2010, a permis de compléter la documentation par des enquêtes orales, la prise de points GPS et l'observation au sol des puits de creusement et d'aération, des canaux et des bassins. La présentation que nous en ferons est par conséquent fondée uniquement sur des observations de surface, aucune fouille archéologique n'ayant été effectuée sur ces installations ${ }^{70}$. La remise en usage, à partir de la première moitié $\mathrm{du} 2 \mathrm{O}^{\mathrm{e}}$ siècle, du réseau hydraulique ancien, si elle permet de mieux suivre sur le terrain les tracés des galeries, perturbe aussi la vision d'ensemble qu' on peut en avoir dans la mesure où les qanats n'ont pas toutes été réutilisées et, lorsqu' elles l'ont été, ce n' est pas nécessairement sur la totalité de leur parcours $^{71}$.

69 Nous remercions la Direction Générale des Antiquités et des Musées de Syrie qui nous a permis d' accéder à cette couverture photographique aérienne et nous a autorisés à réaliser des vues d' hélicoptère, lors d' un survol de la région, le 22 avril 1997.

70 Une qanat a été curée lors de sa remise en fonction dans les années 1990, à Shallāla Ṣaghīra (Joshka Wessels, "Tunneling for survival: ICARDA, Aleppo (Syria)," ICARDA Caravan 11 (1999): 12-13; Joshka Wessels, "Reviving Ancient Water Tunnels in the Desert - Digging for Gold?" Journal of Mountain Science 2, no. 4 (2005): 294-305). Notre connaissance du système hydraulique s' est affinée depuis le début du programme de prospection et notre interprétation de ses différentes composantes a évolué en conséquence par rapport aux premiers articles que nous avons consacrés à ce sujet (cf. Geyer et Rousset, "Les steppes arides"). À noter que les résultats auxquels nous sommes parvenus sont sensiblement différents de ceux publiés par Marlia Mundell Mango pour la région d'Andarīn ("Baths, Reservoirs and Water Use").

71 Les qanats ont progressivement été remises en état à partir de la première moitié du $2 \mathrm{O}^{\mathrm{e}}$ siècle, mais la plupart d' entre elles se sont asséchées dans les années 1960-1970 (Mohamed al-Dbiyat, "Eau et peuplement dans les marges arides: le cas de la région de Salamya en Syrie centrale," dans Stratégies d'acquisition de l'eau et société au Moyen-Orient depuis l'Antiquité, éds. Mohamed al-Dbiyat et Michel Mouton (Beyrouth: Presses de l'Ifpo, 2009), 179-187). La réutilisation est maximale dans la région d'Andarīn à partir des années $195^{\circ}$ (Ronald Jaubert, Mohamed al-Dbiyat et Françoise Debaine, "Transformations des régions cultivées: différenciation des exploitations et extension de l'irrigation," dans Les marges arides du Croissant fertile: peuplements, exploitation et contrôle des ressources en Syrie du Nord, éds. Ronald Jaubert et Bernard Geyer (Lyon: Maison de l'Orient et de la Méditerrannée, 2006), 167-187; Ronald Jaubert et al., "L'exploitation des eaux de subsurface des marges arides de Syrie: dégradation ou transformation?" Sécheresse 13, no. 1 (2002): $43^{-5}$ ). Les auteurs estiment que l'extension des surfaces irriguées à cette époque était probablement comparable à celle de l'époque byzantine. 


\subsection{Les galeries drainantes}

Les galeries drainantes, orientées sud-nord, exploitent les eaux d'inféroflux s'écoulant dans les vallées qui incisent les glacis de piémont des Palmyrénides (figure 6.7). Elles sont nettement plus nombreuses que les qanats issues des tertres de source (nous en avons compté 23). Elles sont pour la plupart localisées sur les quatre oueds majeurs, qui drainent toute la partie centrale de la région, du Jabal Bal'as, au sud-est, jusqu' à la Sabkha al-Ḥarāyik, qui prolonge au nord la grande fayda centrale ${ }^{72}$. Trois ensembles de galeries sont étagés le long de ce réseau hydrographique. Sauf exception, les qanats suivent le lit des oueds, ce qui leur donne un tracé plus ou moins sinueux selon l' encaissement du cours d' eau - de manière générale, les galeries les plus méridionales sont les plus tortueuses.

L' ensemble sud, le plus élevé, se présente au premier abord sous la forme de galeries relativement courtes. Cependant, une étude attentive des photographies aériennes anciennes et de la répartition des bassins, réservoirs construits ou birkats (dépressions non maçonnées), localisés dans la partie aval de ce réseau, permet de supposer que certaines des qanats de ce secteur devaient être à l'origine plus longues. L' exploitation moderne paraît n'avoir tiré parti que de certains segments. On doit probablement la complexité du réseau dans ce secteur à l'intensité de la réutilisation mais aussi au creusement de nouvelles qanats, comme à Shaykh Hlāl. Les sites sont relativement nombreux dans cette zone, notamment dans la partie orientale du secteur. Certains d' entre eux étaient sans doute alimentés en eau par la qanat, comme Umm 'Adasa ${ }^{73}$, sur la Qanāt Kabbāsīn.

L'ensemble intermédiaire est composé de quatre grandes qanats qui alimentent chacune un réservoir, en lisière sud de la grande fayda centrale (figure 6.8).

Les regards suivent le talweg et seules les parties terminales sont à ciel ouvert. C' est le cas de la qanat de Ma'aqar al-Shamālī, un canal de o,70 à $1 \mathrm{~m}$ de largeur, creusé dans la roche, prolonge sur $1 \mathrm{~km}^{74}$. Le conduit de la qanat de Rahjān, qui appartient au même ensemble, à l' est de celle de Umm Miyāl,

72 Cette sabha est une cuvette fermée dont le plancher est tapissé d' alluvions riches en gypse et en chlorure de sodium. Les possibilités de mise en valeur agricole y sont nulles, hormis une éventuelle exploitation du sel (Besançon et Geyer, "Contraintes écogéographiques," $32)$.

73 Le site $95 / 05$, relativement étendu $(700 \times 300 \mathrm{~m})$, est occupé aux périodes romaine (site à cavités), byzantine, omeyyade et abbasside (grand bâtiment carré, d' environ $40 \mathrm{~m}$ de côté, au nord du site).

74 Rousset, "Qanats de la steppe," 252, fig. 8. 


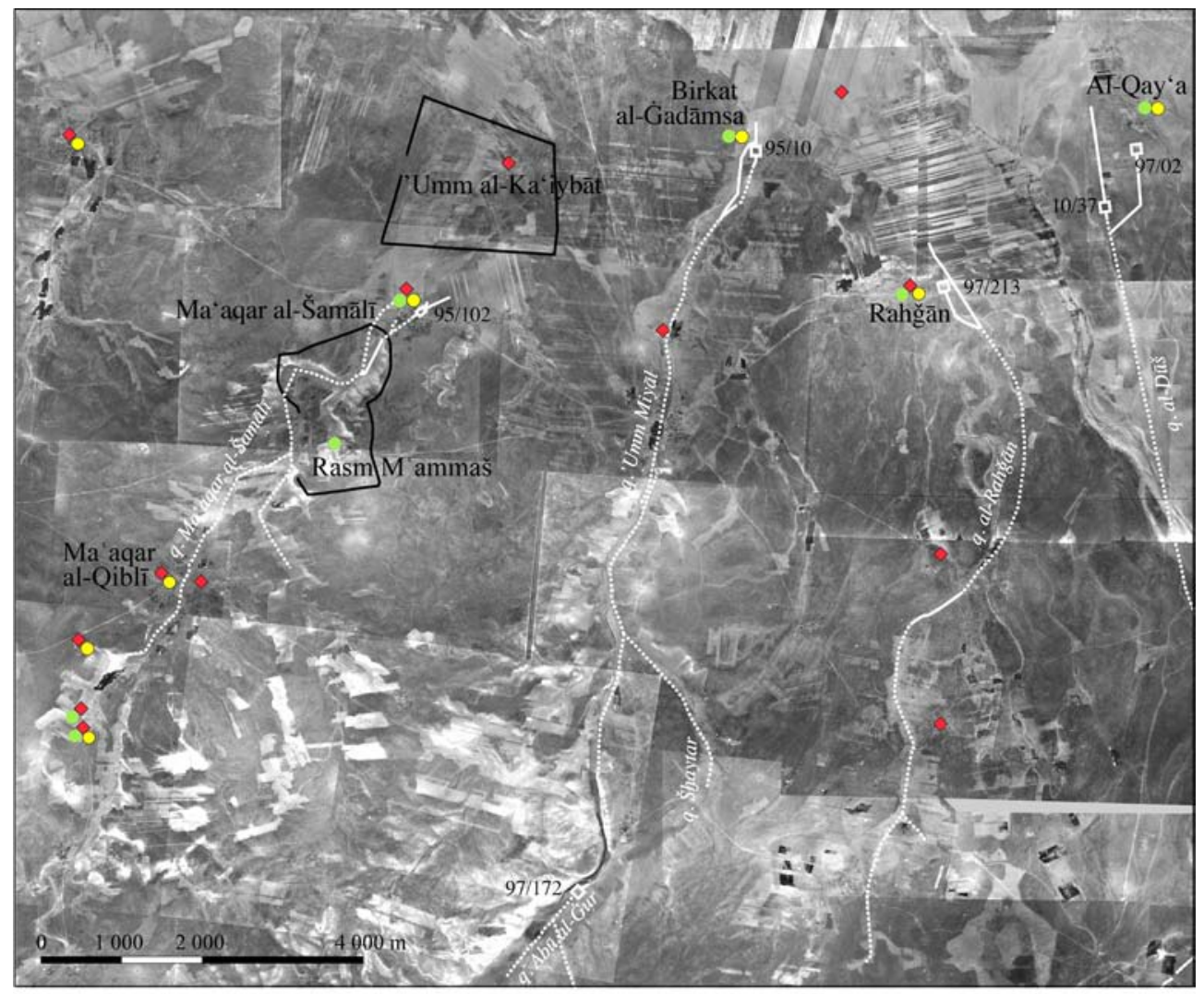

- Site occupé à la période byzantine

- Site occupé à la période omeyyade

- Site occupé à la période abbasside
- Enclos de territoire

Photographie aérienne de 1961. Galerie drainante

Canal

Equidistance des courbes de niveau : $25 \mathrm{~m}$.

FIGURE 6.8 Les galeries drainantes de la zone centrale MARION RIVOAL, MARIE-ODILE ROUSSET (CMISSION MARGES ARIDES 2011

présente une section d'environ $0,50 \mathrm{~m}$ de largeur pour une hauteur totale d'environ $0,90 \mathrm{~m}$.

L'ensemble nord rassemble quatre qanats, au sud et à l'ouest de la ville d'Andarīn, qui irriguaient la partie ouest de la fayda centrale ${ }^{75}$. Les deux qanats

75 Cette partie du réseau a été étudiée par Mundell Mango, "Baths, Reservoirs and Water Use," 75-77. Notre analyse de ce secteur fait apparaître plusieurs différences, notamment dans le tracé des qanats, dont certains éléments, non visibles sur les photographies aériennes de 1958, sont plus récents, alors que d'autres, évidents sur cette documentation, ont été effacés depuis par les travaux agricoles modernes. C' est le cas de la Qanāt al-Ṣālhiyya pour 
est, les plus proches d'Andarīn (al-Andarīn 2 et 3), ont été abandonnées les premières, comme l' agglomération elle-même, à la fin de l'époque omeyyade. Ces deux qanats exploitent les mêmes aquifères que celles des deux ensembles précédents. Elles ont pu s' assécher en raison d' une diminution du niveau piézométrique de la nappe d'inféroflux, en grande partie accaparée par les qanats en amont. Leur tracé général est plus simple que celui des deux qanats occidentales, qui ont été utilisées jusqu' au $10^{\mathrm{e}}$ siècle, probablement avec des réaménagements. Ces deux qanats exploitent des affluents de l'oued principal. L'absence de galeries drainantes à l' amont pourrait expliquer qu' elles soient restées en activité plus longtemps.

Un autre oued majeur a été drainé par des qanats: il s'agit du Wādī d'alHirsh, à l'ouest, qui rejoint lui aussi la Sabkha al-Ḥarāyik (figure 6.7). Dans sa partie amont, une branche - celle de Fuayra - est en relation avec un grand site byzantin (97/275, Fūayra 2), non loin duquel subsiste un bassin. Les autres galeries repérées dans ce secteur semblent plutôt le fait de creusements modernes.

Une seule grande qanat exploite la partie aval de l'oued (Qanāt 'Azīziyya). Formée d' une seule galerie, elle aboutit dans un bassin terminal en rive droite de la vallée, sur une terrasse à dalle calcaire, d' où il était possible d'irriguer par gravité. Le site le plus proche, à $1 \mathrm{~km}$ au nord-est, date de l'époque abbasside. Il est implanté sur un interfluve très bas ouvrant au nord vers la vaste plaine d'alHirsh. Un site occupé du $7^{\mathrm{e}}$ au $9^{\mathrm{e}}$ siècle (oo/33o, Nahr al-Usūd), à mi-parcours, pourrait laisser penser que la qanat était à l'origine plus courte et aurait été prolongée dans un second temps.

Une dernière qanat paraît drainer, non un oued majeur, mais le fond de la grande fayda elle-même. Contrairement aux autres galeries drainantes, la Qanāt al-Zbïb, au centre de la fayda, capte l' eau par plusieurs branches largement étalées, dont deux installées sur des oueds secondaires, et l'achemine vers un réservoir situé $2 \mathrm{~km}$ plus loin (figure 6.9).

laquelle certaines prises d' eau n' ont pas été vues par l'équipe britannique, mais aussi de celle d'al-Zbīb, dont la dernière prise indiquée à l'est correspond en fait à une trace de labour sur Google Earth; et de la Qanāt al-Andarīn 1 qui présente non pas un mais deux réservoirs terminaux. Enfin, l' utilisation à l'époque omeyyade de ces structures n' est que suggérée alors que la mission "Marges arides" l' a clairement mise en évidence grâce à la céramique ramassée sur les sites en liaison avec ces réseaux (76, elle est simplement évoquée dans le cas du réservoir sud-ouest d' Al-Andarīn, où l' analyse par Optically Stimulated Luminescence des fours qui ont été construits pour la fabrication du mortier utilisé pour ce bassin les attribue aux huitième-neuvième siècles). 


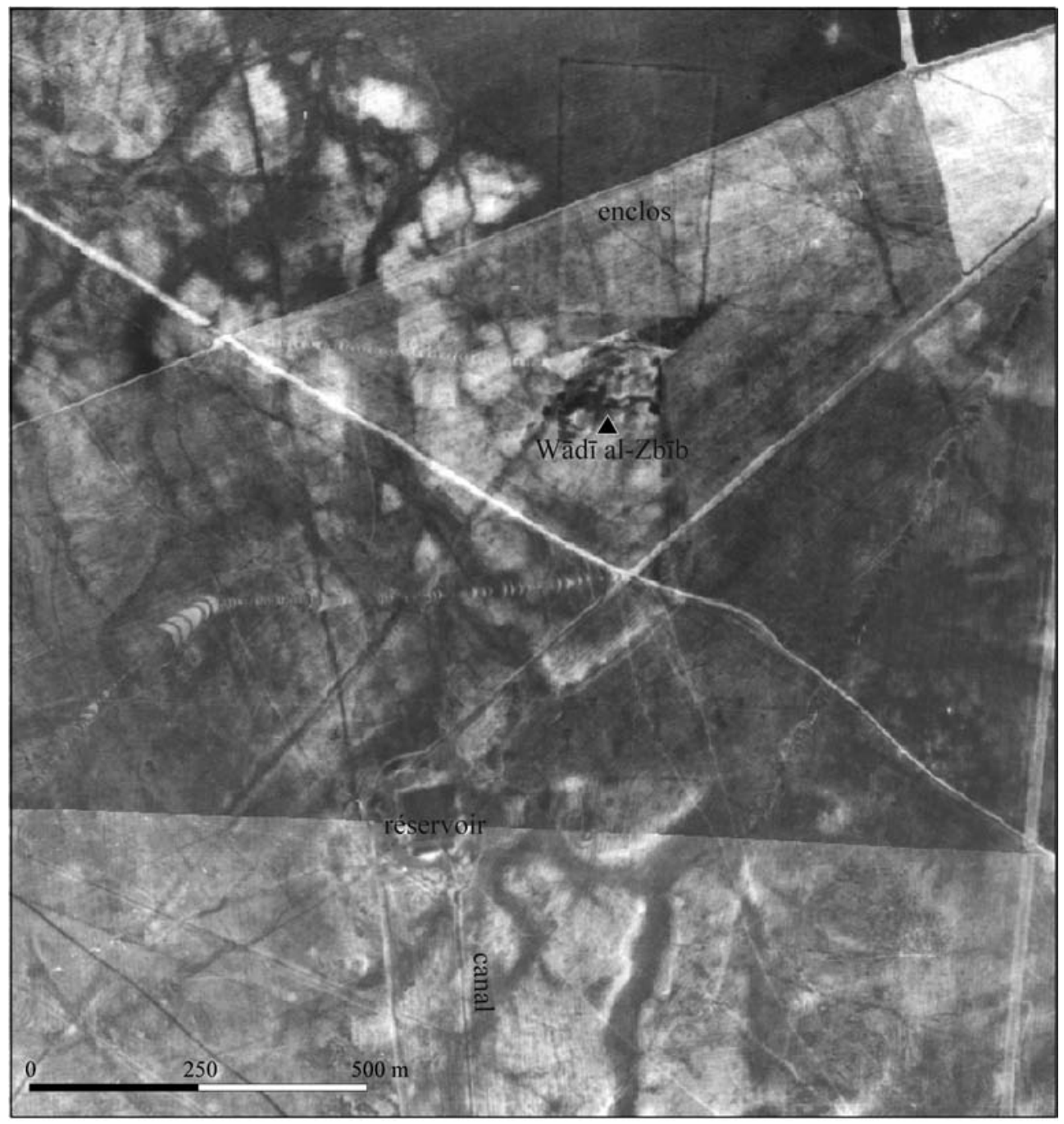

- Site de Wādī al-Zbīỉ (omeyyade)

Photographie aérienne de 1958.

Equidistance des courbes de niveau : $25 \mathrm{~m}$.

FIGURE 6.9 Débouché de la Qanāt al-Zbīb et site de Wādī al-Zbīb 2 MARIE-ODILE ROUSSET 
La fonction de cette qanat pourrait avoir été de collecter l' excédent des eaux d'irrigation déversées par les galeries plus au sud et profondément infiltrées dans l'épaisseur alluviale. La Qanāt al-Zbīb aurait ainsi recueilli ce surplus, inexploitable en l'état pour la culture, et l' aurait redistribué en aval à partir du bassin terminal, un peu plus au nord dans la fayda ${ }^{76}$. Le site de Wādī alZbïb, seul dans les environs du réservoir, est associé à un enclos rectangulaire de $360 \times 220 \mathrm{~m}$, qui devait protéger les cultures irriguées par cette qanat.

\subsection{Les qanats issues des tertres de source}

Ce second groupe de qanats conduit vers l'ouest et vers la grande fayda centrale l'eau captée à partir de tertres de sources artésiennes, concentrés dans la partie centrale de la région ${ }^{77}$. Au moins huit ont été identifiées (figure 6.7). La plus septentrionale est la Qanāt al-Andarīn 1, issue d'un petit tertre de source dans la fayda ${ }^{78}$. La céramique retrouvée sur un bâtiment à côté de l'exutoire indique que la partie terminale aurait été remaniée au cours de la période omeyyade. Un débit important, ou une augmentation de ce débit, aurait entraîné la construction d'un second bassin terminal, plus grand que le premier, à moins qu' on ait eu un besoin en eau plus important. À une période non déterminée, l' eau acheminée par cette qanat est venue s'ajouter par une dérivation à mi-parcours à celle collectée par la Qanāt al-Zbīb, transformant cette dernière en un système mixte.

Les Qanāt 'Ayn al-Nacja et Qanāt 'Ayn Zalīla sont les plus simples. Composées chacune de deux prises d'eau et d'un conduit au tracé quasi rectiligne, elles débouchent dans la grande fayda centrale, sans aménagement particulier. Seule la seconde aboutit à proximité d'un site, une petite ferme occupée aux époques byzantine et omeyyade ${ }^{79}$.

76 Le dénivelé de la pente, sur la longueur de la qanat permet de proposer que la tête pouvait récupérer l' eau à une profondeur maximale de $12 \mathrm{~m}$.

77 Les tertres de sources constituent un ensemble hydrologique particulier (Besançon et Geyer, "Contraintes écogéographiques," 23, fig. 10; Jacques Besançon et al., "Les plateformes gypseuses et les tertres de sources de la région de 'Ayn al-Zarqa (Syrie du Nord)," Bulletin de l'Association de Géographes Français 77, no. 1 (2000): 10-16). On dénombre environ une vingtaine de sources artésiennes, à présent toutes taries du fait de la surexploitation de la nappe phréatique depuis le milieu des années 199o. Les plus grands tertres atteignent vingt mètres de hauteur pour un diamètre d' environ $350 \mathrm{~m}$.

78 Ce tertre est mentionné par Besançon et Geyer, "Contraintes écogéographiques," 23, fig. 10.

79 D'après la base de données de la mission de prospection des Marges arides de la Syrie du Nord; datation d' après la céramique ramassée durant la prospection 2010. 
Deux puits de la Qanāt 'Ayn Zalīla, décapés, laissent voir un conduit vertical, rectangulaire avec son grand axe dans l'axe de la qanat (dimensions: 1,30 m par o,65 m)

Une portion de la Qanāt 'Ayn al-Zarqa, entre les deux bassins 96/1o et 96/11, à l' emplacement où la qanat traverse la dépression périphérique de la plateforme de 'Ayn al-Zarqa, correspond à un passage en aqueduc, aménagé dans un fossé d'environ $4 \mathrm{~m}$ de largeur. La partie médiane est construite et atteint environ $2 \mathrm{~m}$ de large. Sur un radier de petits cailloux, des blocs de gypse sont posés à plat. Ils sont recouverts d' un sol noir, à taches blanchâtres. La conduite pourrait avoir été construite en basalte et blocs de travertin. Un mortier hydraulique lissé, de forme convexe, apparaît en surface.

La Qanāt de Rasm Abū Miyāl comporte deux branches issues de deux sources différentes ('Umm al-Qalaq et al-Shahāțiyya). Cet aménagement est le plus long $(12,5 \mathrm{~km})$ et le plus complexe. Son tracé, assez sinueux est dicté, d' une part, par la nécessité de conserver une pente régulière et, d' autre part, par la volonté de diversifier au maximum les sources d'approvisionnement en eau. Le départ de la canalisation s'effectue en plusieurs points, au sud-est de la plateforme de 'Ayn al-Zarqa, et la qanat s' alimente également à plusieurs sources sur son trajet. Ce système d' adduction comporte des aménagements variés et adaptés aux particularités du terrain: canal souterrain creusé dans la roche, canal à ciel ouvert - dans certains cas, sur un remblai artificiel -, canalisations maçonnées à l'aide d'un mortier hydrofuge charbonneux, digues, bassins de régulation et siphons ${ }^{80}$. Après la jonction avec la Qanat al-Shahạațiyya, une dérivation alimentait une vaste mare, dans l' enceinte de l'enclos de territoire du grand site omeyyade de Rasm Abū Miyāl 1. Le canal se divise ensuite pour, d' une part, se jeter dans un grand réservoir et, d' autre part, irriguer la fayda. Ces qanats sont indissociables de la résidence de Rasm Abū Miyāl 1, assimilable à un «château du désert » : une propriété ceinte de murs, avec un apport d' eau artificiel et des bâtiments secondaires, dont une résidence luxueuse et des constructions plus modestes, de type fermes et/ou maisons ${ }^{81}$. Le site et les aménagements hydrauliques afférents ont été utilisés de manière continue jusqu'à l' époque abbasside ( $9^{\mathrm{e}}$ siècle).

8o Voir Rousset, "Qanats de la steppe," 242-25o.

$81 \quad$ Sur le site de Rasm Abū Miyāl, voir Geyer, "Des fermes byzantines," 119-121; Bernard Geyer et Marie-Odile Rousset, "Rasm Abū Miyāl, château du désert," dans Une aventure archéologique: Antoine Poidebard, photographe et aviateur, éds. Fabrice Denise et Lévon Nordiguian (Marseille: Parenthèses, 2004), 294-295; Genequand et Rousset, "Résidences aristocratiques," 233-248. 
En dehors de la Qanāt Wādī al-Zbīb, un autre système mixte a pu être observé. Il s' agit de la Qanat al-Khafiyya, qui capte l'eau du tertre de source d'al-Shahậțiyya et la conduit vers le Wādī al-'Azīb, qu' elle draine ensuite sur environ $4 \mathrm{~km}$ pour aller irriguer la fayda d' Abū Laffa, à l'ouest, par un canal à ciel ouvert embranché sur un bassin à al-Khafiyya.

\subsection{Les bassins-réservoirs: formes et usages}

Les bassins sont généralement situés dans la partie terminale des qanats, entre le conduit principal et le ou les canaux répartiteurs. Cependant, deux cas présentent des bassins sur le tracé de la canalisation principale. Deux paires de bassins de régulation intermédiaires ont ainsi été repérés sur le tracé des Qanat 'Ayn al-Zarqa et Umm al-Qalaq. Ils sont de petite taille, ronds $(16-17 \mathrm{~m}$ de diamètre pour $97 / 38$ et $97 / 39$, 3o $\mathrm{m}$ de diamètre pour $96 / 11)$ ou carrés $(12 \times 13 \mathrm{~m}$ pour 96/10). Des vestiges d'un habitat semi-sédentaire d'époque omeyyade, à proximité du bassin 96/10, indiquent qu' il a vraisemblablement dû servir également à l' alimentation en eau des éleveurs et de leur bétail. Les puits de qanat qui recoupent le bassin 96/11 montrent un recreusement du canal d' origine et l'abandon de ce bassin, probablement dès l'époque omeyyade.

Les grands réservoirs terminaux sont en général maçonnés. Dans le cas contraire, ce sont des birkats, des dépressions creusées dont le fond et les parois sont recouverts d' argile. Les dimensions dont nous disposons pour les installations de stockage du réseau sud sont variées alors que tous les bassins terminaux des qanats issues des tertres de source mesurent environ $40 \times 40 \mathrm{~m}^{82}$. Il est difficile d'établir des comparaisons fondées sur la taille des bassins-réservoirs, dans la mesure où les dimensions ne sont pas homogènes et où les mesures n' ont pas toujours été prises dans les mêmes conditions, lorsqu' il ne s' agit pas d'estimations. Néanmoins, les variations de la capacité des bassins que suggèrent ces mesures pourraient être liées soit au débit des galeries, lui-même conditionné par des modes d'alimentation différents, soit à l'extension des cultures à irriguer. Le débit des qanats issues des tertres de source serait ainsi moindre que celui des qanats du réseau sud.

L'un des réservoirs les mieux conservés est celui de Ma'aqar al-Shamālī. Ce grand bassin, qui jouxte le village actuel, est installé sur un promontoire, sur un lambeau de glacis à dalle calcaire qui domine une vallée large à fond plat.

82 On peut d' ailleurs rattacher à cet ensemble la qanat 'Amšaradda, au sud-est de la zone prospectée, qui aboutit dans un réservoir de $40 \mathrm{~m}$ de côté (d' après les photos aériennes). Soubhi Mazloum donne pour la birkat 'Amsharadda un diamètre de $40 \mathrm{~m}$ (Mouterde et Poidebard, Le Limes de Chalcis, 1:119). Cependant, les images de Google Earth montrent clairement qu'il s' agit d'un bassin carré. 
Cette disposition est idéale pour un début d'irrigation. De plan rectangulaire $(54,75 \times 39,62 \mathrm{~m}$ pour $3 \mathrm{~m}$ de profondeur $)$, il est orienté au nord-est, c' est-à-dire dans l'axe du débouché de la qanat ${ }^{83}$. Ses forme et taille sont plutôt inhabituelles pour la région puisqu' un seul autre bassin franchement rectangulaire a été observé, à Qanāt al-Andarīn 1 (96/78, 122×103 m). L' eau s'y déversait par un canal taillé dans la roche au milieu de l'un des petits côtés. Elle s'écoulait ensuite à l'opposé, par un conduit aménagé dans le tiers supérieur de la paroi. La base de ce mur ayant été reconstruite récemment, rien ne permet de voir si une autre sortie d'eau, plus basse, avait été prévue à l'origine. Les murs et le sol sont faits de blocs calcaires parfaitement taillés, assemblés à joints vifs $(1 \times 0,40 \mathrm{~m}$ de haut $)$.

Le réservoir de Birkat al-Ghadāmsa (95/10, environ $65 \mathrm{~m}$ de côté), creusé dans la dalle calcaire, est pourvu d' une colonne en basalte qui devait permettre de mesurer le niveau de l' eau ${ }^{84}$.

Deux réservoirs, au sud-ouest (97/122) et au nord-ouest (96/80) de l'agglomération d'Andarīn, ont été étudiés par la mission britannique. Contemporains, ils mesurent chacun $61 \times 61 \mathrm{~m}$ de côté pour 2,50 à $3 \mathrm{~m}$ de profondeur et sont construits en blocs calcaires taillés, avec un sol de galets ou de dalles. Ils sont tous deux richement décorés: niches et colonnettes à motifs gravés pour 97/122, frise ornementale pour 96/80, avec notamment un panneau représentant Jonas et la Baleine. La datation par $\mathrm{C}_{14}$ des charbons de bois inclus dans le mortier du sol du bassin sud-ouest permet de situer sa construction entre 540 et 670 , soit dans le dernier siècle de la domination byzantine ou au début de la période islamique. Des fours à chaux pour la préparation de mortier ont été retrouvés à proximité du bassin 97/122. Les analyses par thermoluminescence les datent des $8^{\mathrm{e}}-9^{\mathrm{e}}$ siècles ${ }^{85}$.

Ces réservoirs sont manifestement à la fois des bassins de régulation et des installations de stockage de l' eau destinée à l' irrigation - c' est ce que suggère la présence d'un canal terminal, en sortie du bassin. Mais les réservoirs d'Andarīn pourraient avoir eu d' autres finalités, pas nécessairement incompatibles avec la première, comme celle de bassin d'élevage pour les poissons ${ }^{86}$. Mais cela supposerait soit la présence de grille ou de tout autre système évitant la fuite des poissons par le canal d'évacuation, soit que le bassin ne servait pas simul-

83 Rousset, "Qanats de la steppe," 253-254, fig. 9 et 10; Genequand et Rousset, "Résidences aristocratiques," 252-256.

84 Genequand et Rousset, "Résidences aristocratiques," 248-252.

85 Mundell Mango, "Baths, Reservoirs and Water Use," 76-79.

86 Marlia Mundell Mango, "Fishing in the Desert," dans Golden Gate, Festchrift for Ihor Ševcenko, éds. Peter Schneider et Olga Strakhov, Palaeoslavica 10, no. 1 (2002): 323-330. 
tanément pour l'irrigation et la pisciculture ${ }^{87}$. Deux canaux de dérivation, en amont du réservoir 88 , pourraient avoir été utilisés pour une irrigation directe, c' est-à-dire sans que l' eau acheminée par la qanat ne transite par le bassin de régulation lorsqu' on destinait ce réservoir à la pisciculture. L'un des canaux le contourne par le sud et aboutit à un réservoir plus petit $(25,60 \times 27,20 \mathrm{~m})$, entièrement construit avec des blocs de remplois, lors d' une réutilisation tardive du système hydraulique. Un autre grand canal terminal, raccordé à ce petit bassin, se dirige vers le sud-ouest sur une distance de $75^{2} \mathrm{~m}$.

La présence des décors sculptés suggère aussi que ces bassins pourraient avoir été utilisés pour des spectacles nautiques ${ }^{89}$. Se pose alors la question de la contemporanéité de ces fonctions, très différentes les unes des autres. Les prospecteurs des Marges arides ont reconnu presque systématiquement, à proximité des bassins terminaux localisés dans ou sur le pourtour de la grande fayda centrale, une construction rectangulaire ou carrée, d' une cinquantaine de mètres de côté voire davantage qui pourrait avoir été une grosse ferme ou un édifice au caractère résidentiel plus prononcé, associé au bassin. La céramique byzantine est très faiblement représentée sur ces sites et seuls quelques bassins ont livré des tessons de cette période. Au contraire, la céramique omeyyade et des fragments de placage de marbre - a été systématiquement retrouvée sur ces bâtiments. La céramique abbasside, quant à elle, n' est présente que sur les édifices au débouché des qanats du réseau sud et sur le bâtiment $\mathrm{A}$ du site de Rasm Abū Miyāl $1^{90}$.

Les indications chronologiques dont nous disposons pour dater ces bassins terminaux et leur utilisation permettent d'envisager des fonctions différentes et susceptibles d'avoir évolué au cours du temps. Nous supposons qu'ils ont été construits en même temps que les galeries drainantes, avant tout dans un but d'irrigation. Pour le réseau qui exploite les sources artésiennes des plateformes gypseuses, pérennes et au débit constant, la fonction première des bassins terminaux serait celle de bassins répartiteurs, c' est-à-dire que leur rôle serait d' abord de redistribuer l' eau vers les terres à irriguer. Dans le cas des galeries qui drainent les nappes d'inféroflux, dont le débit, également pérenne mais

87 D'autre part, les poissons qui auraient été élevés dans ce type de bassin (poisson-chat) peuvent survivre dans très peu d'eau ou dans des eaux peu oxygénées. Leur élevage n'impose donc pas nécessairement de maintenir une circulation d' eau dans les bassins. Mundell Mango, "Baths, Reservoirs and Water Use," 87, fig. 11 et 12. À Ma'aqar al-Shamālī également, un canal étroit est creusé dans la roche, à faible distance de l'entrée de la conduite principale dans le bassin (Rousset, "Qanats de la steppe," 253, fig. 9).

89 Mundell Mango, "Fishing," 325.

$90 \quad$ Rousset, "Qanats de la steppe," 247, fig. 4; Genequand et Rousset, "Résidences aristocratiques," 233. Ce bâtiment est particulier car il est entouré d' un mur d' enclos. 
plus irrégulier - qu' il soit supérieur ou moindre par rapport à celui des qanats en provenance des tertres -, fluctue d'une saison à l' autre, les bassins auraient plutôt eu pour fonction d'emmagasiner l' eau pour en réguler les quantités disponibles pour l' irrigation ou pour abreuver le bétail. À ces usages agricoles s'ajoutent ou succèdent une fonction piscicole et une fonction d'agrément, attestée ailleurs à l'époque omeyyade ${ }^{91}$.

La réutilisation contemporaine des réseaux d'irrigation a laissé de côté plusieurs de ces bassins, comme à Buwaydar 2 (10/20), où de nouveaux puits d' aération recoupent les déblais de creusement du réservoir. À Birkat al-Ghadāmsa, une canalisation à ciel ouvert contourne le bassin pour rejoindre directement le canal répartiteur. À l' extrémité de la Qanat al-'Azīziyya, une conduite moderne est raccordée au tracé d' origine, en amont du bassin, et le contourne par l'est. À Qanat Umm al-Qalaq, un canal secondaire, qui se ramifie ensuite en deux branches, part de la canalisation principale, bien en amont du bassin, pour irriguer la zone située au sud de ce dernier ${ }^{92}$. Dans ce dernier cas, on peut s'interroger sur la contemporanéité des différents aménagements. L' abandon du bassin peut s' expliquer dans certains cas par la difficulté que représente la remise en service des installations anciennes, comme à Ma'aqar al-Shamālī, où le bassin antique a été curé, puis en partie ré-enduit, sans qu'il ait jamais pu retenir l' eau (d'après les témoignages recueillis sur le terrain).

\subsection{Les canaux répartiteurs}

Les canaux répartiteurs qui conduisent l' eau vers les terres à irriguer sont le plus souvent raccordés aux réservoirs terminaux, qui servent alors à réguler les flux et les quantités distribuées. Le canal principal est le plus souvent raccordé au bassin dans l' axe du conduit d'arrivée d'eau, comme à Qanat 'Aziziyya, Qanat Umm al-Qalaq, Qanat 'Ayn al-Zarqa, Qanat Umm Miyāl, Qanat alAndarīn 2 et Qanat al-Andarīn $3^{93}$. Plus rarement, le canal se ramifie à la sortie du bassin en deux branches à $45^{\circ}$ (Qanat Ma'aqar al-Shamālī) ${ }^{94}$. En fonction de la topographie et de la nécessité, l' eau peut parfois être acheminée également par une ou deux autres conduites répartitrices, embranchées perpendiculaire-

91 Denis Genequand, "Économie de production, affirmation du pouvoir et dolce vita: aspects de la politique de l' eau sous les Omeyyades au Bilad al-Sham," dans Stratégies d'acquisition de l'eau et société au Moyen-Orient depuis l'Antiquité, éds. Mohamed al-Dbiyat et Michel Mouton (Beyrouth: Presses de l' Ifpo, 2009), 157-177; Denis Genequand, Les établissements des élites omeyyades en Palmyrène et au Proche-Orient, Bibliothèque Archéologique et Historique 200 (Beyrouth: Presses de l'Ifpo, 2012), 282.

92 Rousset, "Qanats de la steppe," 247, 262, fig. 4 et 15.

93 Rousset, figs 2, 4 et 15; Mundell Mango, "Baths, Reservoirs and Water Use," 87, fig. 11 et 12.

94 Rousset, “Qanats de la steppe," 253, fig. 9. 
ment aux autres côtés du bassin (Qanat Umm al-Qalaq) ${ }^{95}$. La fouille des bassins d'Andarīn a mis en évidence la présence d' écluses à porte pour les canaux latéraux. Le canal axial qui quitte le bassin nord-ouest d' Andarīn (bassin 96/80), en pierre, est connecté au réservoir par un trou au niveau du sol, situé à l' aplomb d' un autre conduit, qui évacue le trop-plein d' eau. Le canal répartiteur a pu être suivi sur près d'un kilomètre, en direction du nord-ouest. Le conduit supérieur, quant à lui, oblique en diagonale vers le nord-est pour irriguer les champs environnants ${ }^{96}$.

Lorsqu' aucun réservoir terminal n'a été repéré, l'extrémité du canal débouche directement au milieu des terres cultivables.

\section{5 «Les maîtres de l'eau» et l'évolution des grands ouvrages hydrauliques: de l'utile à l'agrément}

L'aménagement des qanats est une entreprise délicate qui a été décrite dans les ouvrages d'agronomie anciens ${ }^{97}$. Elle nécessite l'intervention de spécialistes, regroupés en corporations, à différentes étapes de sa réalisation: captage, calcul des altitudes - essentiel, la galerie devant suivre une pente légère et régulière -, creusement, appréciation des débits ... Par conséquent, il est impensable que les qanats aient été construites par les cultivateurs eux-mêmes. Leur usage est soumis à des contraintes juridiques, portant sur le statut des terres et la gestion des tours d' eau. À l'époque abbasside (aux environs de 830), les traités d' agriculture et d'hydraulique évoquent largement la question des droits d'accès à l' eau. Le harìm - une zone d'environ $250 \mathrm{~m}$ de large de part et d'autre de la qanat - est un espace réservé au sein duquel il est interdit de construire une autre qanat, de creuser un puits mais également de cultiver et de bâtir, pour qu' aucun prélèvement ni aucune pollution n' affecte la ressource

95 Rousset, 247, fig. 4; Geyer, Besançon et Rousset, "Les peuplements anciens," 68, fig. 7 .

96 Mundell Mango, "Baths, Reservoirs and Water Use," 87, fig. 12.

97 Nombre de ces écrits s'inspirent de l'Agriculture nabatéenne de Kūtzami, traduite du syriaque vers l' arabe par Ibn Waḩšiyya au $10^{\mathrm{e}}$ siècle, qui expose les techniques de culture et d'irrigation en usage aux $3^{\mathrm{e}-6^{\mathrm{e}}}$ siècles dans le Bilād al-Šām: Simone Zakri, "Pratiques hydroagricoles traditionnelles d'après les manuscrits arabes d'agronomie à la période médiévale dans le monde arabo-musulman," dans Techniques et pratiques hydro-agricoles traditionnelles en domaine irrigué, approche pluridisciplinaire des modes de culture avant

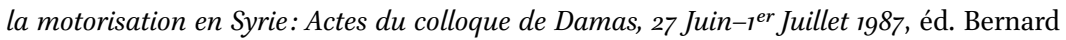
Geyer (Paris: Geuthner, 199o), 37; Patrick Landry, "Eaux souterraines et qanats d' après un livre arabe $\mathrm{du} \mathrm{XI}^{\mathrm{e}}$ siècle," dans Techniques et pratiques hydro-agricoles traditionnelles en domaine irrigué, approche pluridisciplinaire des modes de culture avant la motorisation en Syrie: Actes du colloque de Damas, 27 Juin-1 er Juillet 1987, éd. Bernard Geyer (Paris: Geuthner, 199o), 271-283; Mohamed El-Faiz, Les Maîtres de l'eau: histoire de l'hydraulique arabe (Arles: Actes Sud, 2005). 
exploitée par la qanat ${ }^{98}$. L' existence des qanats implique donc celle d' une autorité en mesure de faire respecter ces contraintes.

Nous l' avons déjà souligné, il est extrêmement difficile, en l' absence de fouilles, de dater ces réseaux d' irrigation. Les renseignements fournis par les textes pour cette région sont extrêmement rares. Une inscription sur un linteau d'époque byzantine, répertoriée dans la région, pourrait faire référence à une qanat entre al-Ḥammām et Khanāṣir (soit une distance d' environ $17 \mathrm{~km}$ ) ${ }^{99}$. Elle mentionne un certain Grègorios Abimenos, également connu pour avoir restauré les portes et l'enceinte de Khanāșir en $604^{100}$. Cependant, bien que la présence d'un tertre de source à $2,5 \mathrm{~km}$ au sud-est du site d' al-Ḥammām soit une bonne configuration pour un départ de qanat, aucune galerie n'a été repérée dans ce secteur, ni sur le terrain, ni sur les photographies aériennes. Depuis la source située au sud d'al-Ḥammām part un canal à ciel ouvert qui se dirige vers le site, sur $800 \mathrm{~m}$, et qui a été recreusé au moins une fois; cependant il est très probablement moderne.

Avec les seules données de la prospection, il est souvent impossible de déterminer la chronologie relative entre les différents segments des conduites et les sites localisés à proximité. De plus, les céramiques sont généralement absentes dans les canaux et rares auprès des bassins. Cependant, l'évolution de la répartition générale des sites d'une période à l'autre livre quelques indices sur l'utilisation du réseau hydraulique. Les sites ont sans doute eu, à un moment donné, un rapport direct avec l' exploitation et la gestion de l' eau. Les implantations romaines, souvent modestes, sont assez régulièrement réparties sur

98 El-Faiz, 175 .

99 Mouterde et Poidebard, Le Limes de Chalcis, 1:207-208: "Il est naturel à cet univers de dérouler [les plaines] après les sommets et [de faire succéder] le sec à l'humide: par un bon vouloir divin, échut à cette source, sortie du roc, la vertu d' exhaler des vapeurs, offrant un remède salutaire convenable aux passants [m. à m. à ceux qui s' arrêtent]. Ayant découvert un canal abondant, Grègorios (?) a opéré un captage, qui étendit commodément jusqu'à sa patrie le flot de vie et de sécurité préventif des maladies. Tout le travail a été accompli [l' an ...], la $12^{\mathrm{e}}$ indiction"; voir aussi Jeanne et Louis Robert, "Bulletin épigraphique," Revue des Études Grecques (1946-1947): 357-359, no. 204, et Jeanne et Louis Robert, "Bulletin épigraphique," Revue des Études Grecques (1954): 175, no. 243. Cette traduction de l'inscription laisse entendre la construction par un évergète d'une conduite d'adduction d' eau vers Anasartha (Khanāṣir) à partir de la source d'al-Ḥammām. Mais l'édition du texte et sa traduction par René Mouterde soulèvent de nombreux problèmes et l'inscription ferait plutôt référence à la construction d'un bain qu'à celle d'une qanat (Gatier, “«Grande»," 97 et n. 25; nous remercions Pierre-Louis Gatier pour les informations complémentaires qu' il a bien voulu nous communiquer).

Jalabert, Inscriptions, nos. 281 et 292. 
l'ensemble du secteur, espacées d'environ $2 \mathrm{~km}$ les unes des autres, avec des sites plus étendus, répartis selon un maillage plus lâche. Ils ne semblent pas entretenir de rapport direct avec les qanats et leur sont vraisemblablement antérieurs car les galeries drainent les terrains qu' elles traversent et les rendent, de ce fait, moins propices à la culture en les privant en partie de l'accès aux nappes d'inféroflux.

Les sites byzantins sont installés soit en rapport direct - à proximité de la tête ou au débouché de la qanat - soit à l'écart de la galerie drainante. On ne les trouve que dans de rares cas sur le trajet de la qanat ${ }^{101}$. Lorsque $c^{\prime}$ est le cas, l'eau devait être remontée depuis les puits par des machines élévatoires - c' est du moins ce que suggère la présence de godets sur certains de ces sites. Ces établissements, des fermes le plus souvent, seraient donc contemporains des qanats, vraisemblablement gérées par des communautés villageoises.

Le système a continué à être exploité et entretenu au début de l'époque islamique. Les sites sont alors plus systématiquement localisés au débouché des qanats, exception faite du réseau sud, issu des Palmyrénides. La présence, à partir de l'époque omeyyade, d'un nouveau type de bâtiment, à proximité immédiate des réservoirs terminaux, témoigne vraisemblablement d'une gestion différente des qanats. L'exploitation des qanats et la mise en valeur des terres qu' elles irriguent seraient le fait d' un grand propriétaire, résidant - peutêtre temporairement - à proximité du débouché de la galerie, plutôt que d'une communauté ${ }^{102}$. Les ressources hydrauliques semblent avoir été suffisantes, au

101 Par exemple la Qanat Umm 'Uwayqīla, au sud-est du réseau et à Umm al-Ka'iybāt (Rousset, "Qanats de la steppe," 256-257).

102 Genequand et Rousset, "Résidences aristocratiques," 259-263. Hugh Kennedy, "The Impact of Muslim Rule on the Pattern of Rural Settlement in Syria," dans La Syrie de Byzance à l'Islam VII ${ }^{e}-V_{I I I}{ }^{e}$ siècles: Actes du Colloque International, Lyon-Paris, 11-15 septembre 199o, éds. Pierre Canivet et Jean-Paul Rey-Coquais (Damas: Institut Français d'Études Arabes de Damas, 1992), 295. L'essentiel de la mise en valeur agricole, d'après Hugh Kennedy, aurait porté, à l'époque omeyyade, sur des zones dites marginales, qui n' auraient connu qu' une occupation sporadique auparavant - le cas des Marges arides de la Syrie du Nord a cependant conduit à corriger fortement ce point de vue - et qui ne furent guère réoccupées par la suite. Il n'était pas nécessaire, au début de l'époque islamique, d'acheter des terres dans les zones de marges - au climat semi-aride ou aride pour les mettre en valeur. Le développement de ces secteurs, planifié par des magnats qui résidaient ou non sur place, est en partie lié à la fiscalité de la dynastie régnante. Les élites omeyyades vivaient pour une grande part de leurs propres ressources et non du revenu d'une taxation générale - la perception de l'impôt n' est centralisée qu'à partir de l'époque abbasside. Voir également Michael Morony, "Economic boundaries? Late Antiquity and Early Islam," Journal of the Economic and Social History of the Orient 47, no. 2 (2004): 166-194. 
$9^{\mathrm{e}}$ siècle, pour effectuer des prélèvements en amont sur certaines qanats. Cet apport en eau est parfois complété par des citernes d' appoint, comme à Umm 'Adasa ou Rasm M'ammash ${ }^{103}$.

On peut observer des différences notables dans la chronologie de l'utilisation des ouvrages hydrauliques, d'un secteur à l'autre. Dans la région des tertres de sources, les deux qanats septentrionales pourraient avoir été abandonnées après le $6^{\mathrm{e}}$ siècle alors qu' une qanat plus au sud (Umm al-Qalaq) aurait été réutilisée, modifiée ou bien construite à l'époque omeyyade, en relation avec un établissement de type «château du désert »104. La répartition des sites autour des qanats d'Andarīn semble indiquer que seules les deux qanats occidentales étaient encore - au moins en partie - en activité à l'époque abbasside ( $9^{\mathrm{e}}$ siècle), alors que les plus proches d'Andarīn auraient périclité en même temps que l'agglomération elle-même. Enfin, à l'époque abbasside, l'utilisation des ouvrages hydrauliques apparait variable selon les zones. D'une part, l' extension du réseau en usage se réduit, car les sites de cette période sont presque tous répartis dans la moitié sud-ouest de la région. D' autre part, comme à l'époque omeyyade, des résidences aristocratiques existent à proximité des bassins terminaux. On observe autour de ces établissements des murs d'enceinte, voire dans certains cas des fossés, qui témoigneraient de la volonté de défendre ces installations du fait d'une insécurité grandissante, en partie due à la guerre civile des années 830 . Le caractère défensif de ces établissements est particulièrement bien représenté par le camp fortifié du $9^{\mathrm{e}}$ siècle à Abū al-Khanādiq. Une forteresse de basalte y est entourée de deux fossés, doublés chacun d' un mur en brique crue ${ }^{105}$.

La période byzantine est probablement celle qui a vu l' aménagement de la majeure partie des grands ouvrages hydrauliques, dans le cadre d'une exploi-

103 Site localisé le long de la Qanat Ma'aqar al-Shamālī (Rousset, “Qanats de la steppe," 255256).

104 Genequand et Rousset, "Résidences aristocratiques," 233-248.

105 Marie-Odile Rousset, "Traces of the Banu Salih in the Syrian Steppe? The Fortresses of Qinnasrin and Abu al-Khanadiq," Levant 45 (2013), 69-95; Marie-Odile Rousset, "Deux sites fortifiés au début de l'époque islamique au Bilād al-Šām: Qinnasrīn et Abū al-Hुanādiq," dans La guerre dans le Proche-Orient médiéval ( $X^{e}-X V^{e}$ s.): état de la question, lieux communs, nouvelles approches, éds. Mathieu Eychenne, Stéphane Pradines et Abbès Zouache, Recherches d'archéologie, de philologie et d'histoire 37 (Le Caire: IFAO, 2014), 193-229. Aucune céramique antérieure au début du $9^{\mathrm{e}}$ siècle n' a été retrouvée sur ce site, en grande partie construit avec des matériaux de remploi. Les inscriptions qui y ont été relevées sont selon toute probabilité hors contexte (Griesheimer, "L' occupation byzantine," 141142, no. 25). Abū al-Khanādiq n'est donc vraisemblablement pas l'étape sur l'Itinéraire Antonin, suggérée par Marlia Mundell Mango (Mundell Mango, "Baths, Reservoirs and Water Use," 78). 
tation générale et raisonnée de l'ensemble des ressources de la région. Les réseaux hydrauliques ont dû, d'une part, permettre d'augmenter les rendements des céréales et, d'autre part, favoriser dans l'ouest de la région irriguée le passage de la céréaliculture à l'arboriculture. Les installations ont été entretenues et de nouvelles modalités de gestion de ces aménagements paraissent s' instaurer au début de l'époque islamique. L'époque omeyyade voit l'émergence de grands projets d' aménagements agricoles dans l'ensemble du Bilād al-Shām et les Marges arides de la Syrie du Nord profitent, elles aussi, de ce mouvement ${ }^{106}$. L'importance de cette région pour la famille régnante transparaît dans les textes: on sait qu' en 7o9, le calife omeyyade al-Walīd b. 'Abd al-Malik séjournait à Khunāșira (Khanāșir) ${ }^{107}$. Le calife 'Umar b. 'Abd al'Azīz (717-720) s'établît au même endroit. Il s'y était fait construire un château où il résidait fréquemment; il y mourut en $720^{108}$. Qașțal est mentionné dans les textes comme l'une des résidences du prince al-'Abbās, qui s'y installa en 744, fuyant une épidémie qui ravageait Damas. Son frère, Yazīd b. alWalīd, s'établît un peu plus loin, à Jarūd [?] ${ }^{109}$. En 745, le calife Marwan II passa par Qașțal et Sūriyā (Ithriyā) sur son trajet vers Ruṣāfa ${ }^{110}$. Ainsi qu' en témoignent les vestiges archéologiques (céramiques, ouvrages hydrauliques, résidences ...), cette zone était par conséquent directement contrôlée par la famille omeyyade.

À partir du début de l'époque abbasside, la superficie des zones irriguées a dû diminuer puisque seules les qanats de l' ouest et du sud sont maintenues en activité. L' irrigation serait alors destinée aux cultures à forte valeur ajoutée, comme par exemple celle du safran ${ }^{111}$. Les textes rapportent que Șâlih b. 'Abd Allāh b. al-'Abbās, nommé gouverneur du sud et du centre de la Syrie en $75^{8}$, s'installa à Salamiyya et s'appliqua à reconstruire la ville. Son fils, 'Abd Allāh, poursuivit son entreprise et en profita pour développer le système d' irrigation de l' agglomération et de ses environs ${ }^{112}$. De nombreux membres de la famille

106 Kennedy, "The Impact of Muslim Rule," 293; Genequand, Les établissements; Genequand et Rousset, "Résidences aristocratiques," 233-265.

107 Musil, Palmyrena, 204.

108 al-Ṭabarī, Tārīkh al-rusūl wa-l-mulūk, trad. Hermann Zotenberg (Paris: Maisonneuve, 1958), $2: 1361$.

109 al-Ṭabarī, 1784.

110 al-Ṭabarī, $1896,1908$.

111 Un texte mentionne une culture irriguée du safran dans la région de Salamiyya (André Miquel, La géographie humaine du monde musulman jusqu'au milieu du $n^{e}$ siècle (Paris: École des Hautes Études en Sciences Sociales, 1980), 435).

112 al-Ya'qubi, Les pays, trad. Gaston Wiet, Textes et traductions d'auteurs orientaux I (Le Caire: IfAO, 1937), 324; Rousset, "Traces of the Banu Salih," 91-92. 
abbasside hāshimite vécurent à Salamiyya au début de la période abbasside. Vers le début du $9^{\mathrm{e}}$ siècle, 'Abd Allāh, arrière-petit-fils de l'imam Jáfar al-Ṣādiq et réfugié en Syrie, s'installa à Salamiyya et s'y fit construire un palais somptueux ${ }^{113}$.

L'abandon généralisé des réseaux hydrauliques, au milieu du $9^{\mathrm{e}}$ siècle, fait suite aux tentatives de reconquête byzantine et à la destruction des arbres par les Hamdānides ${ }^{114}$. Le climat d'insécurité qui régnait alors ne permettait plus l'entretien des grands ouvrages et la disparition de l'arboriculture privait la population d'une part importante de ses revenus. Dès lors, le maintien du peuplement dans cette région fut compromis ${ }^{115}$.

\section{Les citernes}

Si les ouvrages hydrauliques évoqués ci-dessus sont en priorité destinés à l'irrigation, une autre catégorie d'aménagement est dévolue plus spécifiquement - mais non exclusivement - à l' approvisionnement en eau des hommes et des animaux. Ce sont les puits et les citernes. Nous nous contenterons d'évoquer les secondes car elles témoignent d'une volonté de définir les parcours des populations nomades en constituant des réserves d' eau dans les secteurs les plus arides.

La citerne est un aménagement particulièrement adapté à la faible dotation pluviométrique et à des milieux où le substrat et les formations superficielles favorisent le ruissellement (affleurement de roches compactes et de dalles cimentées ${ }^{116}$. Son ouverture réduite, souvent creusée dans le calcaire, limite l'évaporation et les risques de pollution. Alimentée par les crues des oueds

113 Johannes Hendrik Kramers et Farhad Daftary, "Salamiyya," dans Encyclopaedia of Islam, 2nd ed. (Leiden: Brill, 1995), $8: 95^{2-955}$.

114 Nicéphore Phocas attaque Qinnasrīn en 961 et ravage douze grandes villes de Syrie du Nord en 968. En 944, au cours de la lutte qui oppose Sayf al-Dawla aux Ikhshidides, les arbres fruitiers autour d'Alep sont sciés et les Alépins contraints d' importer leurs fruits et leurs légumes depuis la région d' Antioche, en territoire byzantin (Thierry Bianquis, "Pouvoirs arabes à Alep aux $\mathrm{X}^{\mathrm{e}}$ et XI ${ }^{\mathrm{e}}$ siècles," Revue des mondes musulmans et de la Méditerranée 62 (1991): 53-54).

115 Geyer et Rousset, "Déterminants géoarchéologiques," 92.

116 Besançon et Geyer, "Contraintes écogéographiques," 41-42; Bernard Geyer, Jacques Besançon et Marie-Odile Rousset, "Les citernes pluviales des steppes syriennes, éléments de la conquête d' une marge aride," dans Habitat et environnement: prospections dans les Marges arides de la Syrie du Nord, Travaux de la Maison de l'Orient 71, Conquête de la steppe IV (Lyon: Maison de l' Orient et de la Méditerranée, 2016). 
ou par le ruissellement qui ravine les pentes immédiatement sus-jacentes, elle capte l' eau de pluie avant qu' elle ne s' infiltre ou ne s'évapore.

La forme de la citerne dépend du substrat dans lequel elle est creusée. Les plus petites sont généralement piriformes. Les grandes sont cubiques ou parallélépipédiques, parfois aménagées immédiatement sous la dalle calcaire qui sert ainsi de couverture, et parfois plus profondes.

La modicité des cumuls annuels de précipitation impose de tirer parti au mieux de l' eau fugitive et, pour ce faire, de multiplier les dispositifs de stockage. À Rasm Kandūš, en plus de celles disséminées dans la zone d'habitat, une cinquantaine de citernes ont été dénombrées dans les alentours proches ${ }^{117}$.

Une fois creusées, les citernes demandent un entretien: curage pour évacuer les sédiments transportés par le ruissellement, qui diminuent progressivement les capacités de stockage, et réfection des enduits. Ces opérations nécessitent une concertation entre les différents utilisateurs.

\subsection{Les citernes associées aux agglomérations: une gestion collective}

Dans certaines grandes agglomérations, comme Dawsa ou Rasm al-Ahmar 3, des citernes ont manifestement été aménagées par une collectivité. La surface et la capacité de ces installations est parfois perceptible par la quantité de déblais qui la jouxte mais pas toujours, les déblais de creusement, blocs etéclats de roche, ayant sans doute été utilisés pour les bâtiments. Dans le cas de Rasm al-Ahmar 3, une agglomération qui s' étend sur $850 \mathrm{~m}$ environ du nord au sud et approximativement $700 \mathrm{~m}$ d' est en ouest, un secteur entier est dévolu au stockage de l' eau: deux groupes de citernes, de plan carré, avec enduit et margelle, sont regroupés dans la partie sud-est, non bâtie, du site. Elles sont installées sur une déclivité très peu marquée, à proximité d'un grand enclos qui aurait servi au parcage des animaux ${ }^{118}$. De grosses buttes-dépotoirs, très sombres, intercalées entre les zones d'habitation pourraient traduire l'importance des activités d'élevage sur ce site. Il pourrait en effet s' agir de tas de fumier provenant du nettoyage de l'enclos ${ }^{119}$.

\footnotetext{
117 Duvette, "Habitat byzantin," 186.

118 Geyer, "Pratiques d' acquisition," 41, fig. 17-18; Geyer et Rousset, "Déterminants géoarchéologiques," 85 ; Rivoal, "Entre steppe et plateaux basaltiques," en part. fig. 9.

119 Marion Rivoal, "Autour des dépotoirs: production, gestion et exploitation des excréments animaux dans la steppe syrienne pendant l'Antiquité tardive," dans Habitat et environnement: prospections dans les Marges arides de la Syrie du Nord, éds. Marie-Odile Rousset, Bernard Geyer, Pierre-Louis Gatier et Nazir Awad, Travaux de la Maison de l'Orient, Conquête de la steppe IV (Lyon: Maison de l'Orient et de la Méditerranée, 2016), 169-201.
} 
Dawsa est une agglomération étendue située dans une zone répulsive, globalement défavorable à la culture. En conséquence, l'économie du site paraît majoritairement tournée vers l'élevage. De nombreuses citernes ont été repérées, tant dans la zone d' habitat que dans celle où se concentrent des enclos qui servent probablement à parquer des troupeaux ${ }^{120}$. Séparés par des allées, ces enclos pourraient être les vestiges d'un lieu de rassemblement du petit bétail (caprinés) et de vente des sous-produits de l'élevage. L'agglomération aurait ainsi fonctionné comme un marché, à la lisière des zones dévolues à l'élevage (zone III) et à la culture, notamment irriguée (zone II) ${ }^{121}$.

À Abū al-Khanādiq, une vingtaine de citernes, de plus de $4 \mathrm{~m}$ de profondeur conservée, a été aménagée dans une zone qui jouxte la forteresse de basalte ${ }^{122}$. Seul exemple de ce type, ces citernes permettaient de constituer des réserves d' eau conséquentes, en cas de siège. Néanmoins, dans ce cas précis, l'eau emmagasinée ne semble pas provenir des écoulements de surface, nécessairement restreints dans une zone peu étendue limitée par deux fossés. Il pourrait s' agir d' une réserve d' eau pluviale, recueillie aux toits du bâtiment voisin, ou d'une quantité d'eau transportée depuis l'extérieur du site. Mais seule une autorité - militaire, dans ce cas - devait permettre d'entretenir et d' approvisionner régulièrement ce dispositif.

\subsection{Les citernes isolées et la fixation des nomades}

Dans l' est de la région, sur le plateau calcaire d' al-'Alanda, dans le secteur de Rasm al-Jaāâr (figure 6.10), une série de citernes isolées a été observée ${ }^{123}$.

Elles sont le plus souvent aménagées dans le lit majeur de l'oued et se remplissent à la faveur du ruissellement, les collines environnantes concentrant les eaux de pluies dans le talweg. Elles sont particulièrement grandes - jusqu'à 17,50 $\mathrm{m}$ de profondeur pour $16 \mathrm{~m}$ de côté - et leur capacité semble avoir imposé de réserver, lors du creusement, des piliers qui soutiennent le plafond. On accédait à ces citernes par un puits étroit, de plusieurs mètres de long pour les plus profondes, qui traversait les strates meubles ou fissurées et s' évasait ensuite en une vaste salle creusée dans la roche saine.

Les déblais de curage de la citerne - plus rarement ceux provenant du creusement - sont disposés en arc de cercle en contrebas ou en aval de l' embou-

\footnotetext{
120 Rousset et Duvette, "L'élevage," 490-491.

121 Geyer et Rousset, "Les steppes arides," 116-117.

122 Rousset, "Deux sites fortifiés," 211 et fig. 15.

123 D' autres groupements de citernes ont été repérés, par exemple dans certains des oueds du Jabal Bal'as dans la région de Umm al-Fuhūd, sur la retombée du massif nord palmyrénien.
} 


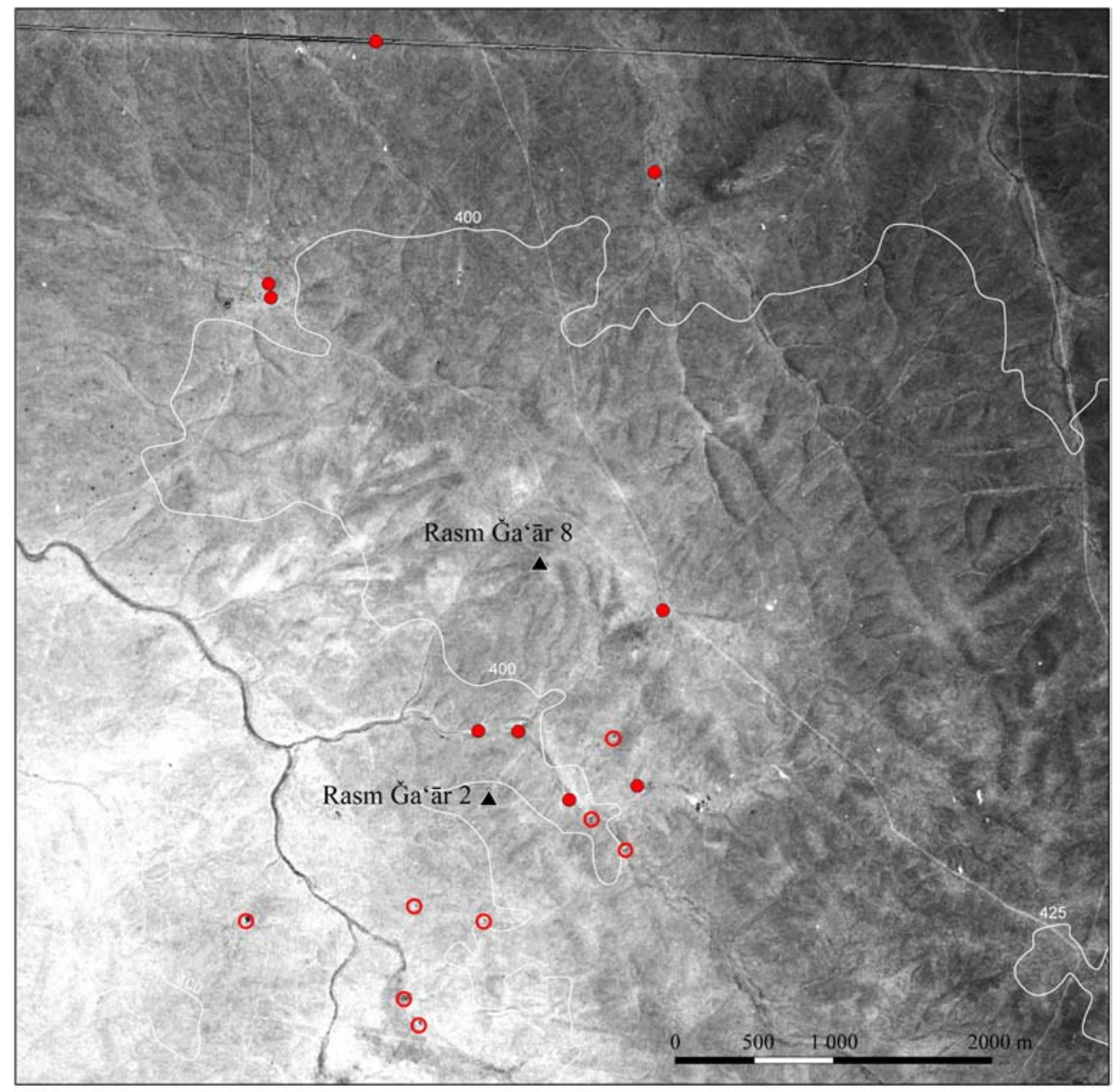

$\Delta$ Site

Photographie aérienne de 1958.

- Citerne isolée vue sur le terrain

○ Citerne isolée repérée sur photographie aérienne

Equidistance des courbes de niveau : $25 \mathrm{~m}$.

FIGURE 6.10 Répartition des citernes dans la région de Rasm Ğacār

MARION RIVOAL, MARIE-ODILE ROUSSET @MISSION MARGES ARIDES

2011

chure, de manière à former une sorte de barrage de retenue qui contribue à une récupération maximale du ruissellement. Des rigoles peuvent également être aménagées pour guider l' eau vers la bouche de la citerne.

Dans ce secteur à l' aridité climatique et édaphique marquée, avec un accès à l'eau problématique sinon inexistant sans aménagement, seul l'élevage est 
praticable. De fait, des traces de campements anciens ont été repérées presque systématiquement non loin de chacune de ces citernes. Ce sont des espaces nettoyés, avec un tas de petites pierres repoussé en périphérie - vestiges des balayages successifs de la zone située à l' avant de la tente - et parfois une butte plus terreuse - peut-être une petite construction en brique crue. Seul le site de Rasm al-Jaār 2 peut être attribué à une occupation de semi-sédentaires, datée de l'époque omeyyade. Il se compose de deux constructions, distantes d'une centaine de mètres, constituées, pour l'une, d' une seule pièce de $5,50 \mathrm{~m}$ de côté et, pour l'autre, de trois pièces accolées, un peu plus grandes $(6 \mathrm{~m}$ de côté). Les deux bâtiments sont construits en blocs de gypse et la largeur des arases de murs $(0,8 \circ \mathrm{m})$ permet de restituer une couverture en coupole. Un autre site, occupé à l'époque byzantine, correspond à un unique petit bâtiment de stockage formé d'une seule pièce de $5 \mathrm{~m}$ de côté (Rasm Ja'ār 8). La base des murs, construite en blocs de gypse et de dalle, soutenait les murs de terre crue.

Les citernes ont rendu possible l' occupation temporaire et parfois permanente dans des zones répulsives. Le nombre de citernes construites dans la région de Rasm al-Jaār et surtout leur capacité laissent penser que ces aménagements ne sont vraisemblablement pas le fait des nomades eux-mêmes mais qu' ils ont été réalisés dans le cadre d' une politique de mise en valeur généralisée de la région. Même si la maîtrise de ces dispositifs de stockage, faciles à mettre en œuvre, est bien antérieure à l'époque byzantine, ce n' est qu'à partir de cette période qu' ils semblent avoir été utilisés comme un moyen de contrôler le peuplement nomade. Le matériel céramique, récolté dans les déblais de curage ou les campements associés aux citernes, bien que peu abondant, indique une première utilisation de ces citernes aux alentours des $5^{\mathrm{e}}-6^{\mathrm{e}}$ siècles, puis tout au long des $7^{\mathrm{e}-8^{\mathrm{e}}}$ siècles et, dans une moindre mesure, aux $9^{\mathrm{e}}-10^{\mathrm{e}}$ siècles. La représentation des différentes périodes, en terme de matériel, est la même dans le secteur de Rasm al-Ja'ār que dans les zones situées plus à l' ouest. Cela signifierait que, entre l'époque byzantine et l'époque abbasside, l'occupation purement nomade évolue dans les mêmes proportions que l'occupation semi-sédentaire ou sédentaire. Le recul du peuplement sédentaire vers l' ouest, auquel on assiste entre le $7^{\mathrm{e}}$ et le $10^{\mathrm{e}}$ siècle, n' est donc vraisemblablement pas à imputer à une modification de la nature du peuplement. Le reflux des sédentaires ne paraît pas coïncider avec une augmentation de la population nomade, ce qui tend à montrer qu'il s'agit bien d'un déclin démographique, touchant les populations sédentaires comme les populations nomades et non d' un changement de stratégie de subsistance des agriculteurs sédentaires, qui se seraient progressivement tournés vers le pastoralisme nomade. La céramique montre également 
une réutilisation tardive des citernes, à l'époque médiévale qui correspond, dans la région, à une période d'élevage intensif, ainsi qu'à l'époque contemporaine.

\section{5}

\section{Conclusion}

Il est difficile, dans l'état actuel de nos connaissances, basées avant tout sur l'archéologie, d' apporter une réponse tranchée et définitive aux diverses interrogations que soulèvent les modalités d'organisation et de contrôle des campagnes des Marges arides de la Syrie du Nord. Les structures agraires, tout comme les aménagements hydrauliques, démontrent la variété des situations: situations économiques, indéniablement, mais probablement aussi politiques, fiscales, administratives et démographiques. Les vestiges agraires et les installations hydrauliques témoignent de changements (notament d'investissements économiques) et d'adaptations dans les stratégies d'exploitation des ressources, tant agricoles qu'hydrologiques. On ne peut dater précisément ces évolutions qui traduisent les efforts et les ambitions des agriculteurs pour rendre une terre productive et la bonifier, si difficile qu'ait pu paraître, de prime abord, la culture dans certains contextes. Car ces stratégies économiques s' inscrivent dans la longue durée, à partir du $3^{\mathrm{e}}$ siècle au plus tard ${ }^{124}$ et jusqu' au milieu du $10^{\mathrm{e}}$ siècle, qui voit se confirmer, pour au moins deux siècles, le recul des sédentaires amorcé dès le $7^{\mathrm{e}}$ siècle ${ }^{125}$.

L'émergence de quelques grands pôles dans la steppe comme Andarīn, avec le réseau de galeries drainantes qui irrigue la grande fayda centrale, et Ithriyā, avec son vaste enclos de territoire - s'ils sont bien contemporains -, pour ne citer que ces deux-là, confirment la pertinence et le succès de ces stratégies. La spécialisation des orientations économiques en fonction des qualités intrinsèques des terroirs, qu' on devine au travers des aménagements agraires et hydrauliques, montre que les exploitants, paysans et éleveurs des Marges arides, ont atteint certains de leurs objectifs et sont parvenus à incorporer leurs productions au marché dynamique qui caractérise la fin de la période byzantine et vraisemblablement aussi le $7^{\mathrm{e}}$ siècle. Ces stratégies économiques impliquent aussi bien les populations de cultivateurs sédentaires que celles des

124 À noter que cette période coïncide également avec les premiers indices que nous avons d'une organisation du territoire dans le Massif calcaire (Georges Tchalenko, Villages antiques de la Syrie du Nord: le massif du Bélus à l'époque romaine (Paris: Geuthner, 19531958), 1:130-131, 3:6-11).

125 Geyer et Rousset, "Déterminants géoarchéologiques," 92. 
éleveurs. À cette dernière catégorie appartiennent les éleveurs sédentaires, non itinérants, avec leurs troupeaux de moutons et de chèvres, mais aussi vraisemblablement les pasteurs nomades. Les citernes construites dans le secteur de Rasm al-Ja'ār démontrent une tentative de contrôle de ces populations et l'on peut raisonnablement supposer que ce contrôle précède et/ou implique une politique d'intégration, notamment économique.

L'évolution des stratégies d'économie agricole mises en évidence par l'analyse des traces matérielles s'effectue en parallèle avec l'évolution de la cité de Chalcis du Bélus / Qinnasrin ${ }^{126}$ et c' est probablement elle qui contrôle cette chôra steppique. Cependant, d'autres formes de structures territoriales ont également pu exercer leur autorité sur ces zones de marges, territoires tribaux ou domaines impériaux ${ }^{127}$. Lyon, 2011.

\section{Bibliographie}

\section{Sources primaires}

al-Bakrī (m. 487/1094). Kitāb al-masālik wa-l-mamālik: juz'ān. Édité par Adrien van Leeuwen et André Ferré. Carthage-Tunis: Bayt al-Hakima Dār al-'Arabiyya lil-Kitāb, 1992.

Corpus Inscriptionum Latinarum, Voluminis tertii: Inscriptiones Asiae provinciarum Europae graecarum Illyrici latinae Pars posterior. Édité par Theodor Mommsen. Berlin: G. Reimer, 1873 .

Ibn Ḥawqal (m. 367/977). Kitäb șürat al-arḍ. Traduit par Johannes Hendrik Kramers et Gaston Wiet. Beyrouth/Paris: al-Laǵna ad-duwalīya li-tarǵamat ar-rawāì' et G.P. Maisonneuve et Larose, 1964.

Notitia dignitatum: Accedunt Notitia urbis Constantinopolitanae et Laterculi provinciarum. Édité par Otto Seeck. Berlin: Berolini, 1876.

al-Ṭabarī (m. 310/923). Tārīkh al-rusūl wa-l-mulük. Traduit par Hermann Zotenberg. 4 vols. Paris: Maisonneuve, 1958.

al-Ya'qubī (m. 284/897). Les pays. Traduit par Gaston Wiet. Textes et traductions d'auteurs orientaux I. Le Caire: IFAO, 1937.

Yāqūt al-Rūmī (m. 626/1229). Kitāb mứğam al-buldān.Édité par Ferdinand Wüstenfeld. 6 vols. Leipzig: F.A. Brockhaus, 1866-1873.

126 Rousset, "De Chalcis à Qinnasrin," 311-340.

127 Gatier, “«Grande»," 105 . 


\section{Sources secondaires}

Besançon, Jacques et Bernard Geyer. "Contraintes écogéographiques et modes d'occupation du sol." Dans Les marges arides du croissant fertile: peuplements, exploitation et contrôle des ressources en Syrie du Nord, édité par Ronald Jaubert et Bernard Geyer, 11-53. Lyon: Maison de l' Orient et de la Méditerranée, 2006.

Besançon, Jacques, Bernard Geyer, Sultan Muhesen et Marie-Odile Rousset. "Les plateformes gypseuses et les tertres de sources de la région de 'Ayn al-Zarqa (Syrie du Nord)." Bulletin de l'Association de Géographes Français 77, no. 1 (2000):10-16.

Bianquis, Thierry. "Pouvoirs arabes à Alep aux $\mathrm{X}^{\mathrm{e}}$ et XI ${ }^{\mathrm{e}}$ siècles." Revue des mondes musulmans et de la Méditerranée 62 (1991): 49-59.

Blanchemanche, Philippe. Bâtisseurs de paysages: terrassement, épierrement et petite hydraulique agricole en Europe, XVII ${ }^{e}-X I X^{e}$ siècles. Paris: Maison des Sciences de l'Homme, 1990.

Boissière, Thierry. Le jardinier et le citadin: ethnologie d'un espace agricole urbain dans la vallée de l'Oronte en Syrie. Damas: Institut Français du Proche-Orient, 2005.

Braemer, Franck, Bernard Geyer, Corinne Castel et Maamoun Abdulkarim. "Conquest of New Lands and Water Systems in the Western Fertile Crescent (Central and Southern Syria)." Water History 2 (2010): 91-114.

Butler, Howard Crosby. Syria: Publications of an American Archaeological Expedition to Syria in 1899-1900, vol. 2, Architecture and Other Arts. New York: The Century Company, 1903 .

Butler, Howard Crosby. Syria: Publications of the Princeton University Archaeological Expeditions to Syria in 1904-1905 and 1909, vol. 2, Architecture, Section B, Northern Syria. Leyde: Brill, 1920.

Callot, Olivier. "Monnaies trouvées dans les Marges arides (Syrie)." Dans Entre nomades et sédentaires: prospections en Syrie du Nord et en Jordanie du Sud, édité par PierreLouis Gatier, Bernard Geyer et Marie-Odile Rousset, 271-288. Lyon: Maison de l' Orient et de la Méditerranée, 2010.

al-Dbiyat, Mohamed. "Eau et peuplement dans les marges arides: le cas de la région de Salamya en Syrie centrale." Dans Stratégies d'acquisition de l'eau et société au MoyenOrient depuis l'Antiquité, édité par Mohamed al-Dbiyat et Michel Mouton, 179-187. Beyrouth: Presses de l' Ifpo, 2009.

Dussaud, René. Topographie historique de la Syrie antique et médiévale. Paris: Geuthner, 1927.

Duvette, Catherine. "Habitat byzantin dans la steppe: maisons et villages de terre." Dans Entre nomades et sédentaires. Prospections en Syrie du Nord et en Jordanie du Sud, édité par Pierre-Louis Gatier, Bernard Geyer et Marie-Odile Rousset, 175-207. Lyon: Maison de l' Orient et de la Méditerranée, 2010.

Duvette, Catherine. "Rasm Afandi, villge des steppes de Syrie." Dans Habitat et environnement: prospections dans les Marges arides de la Syrie du Nord, édité par M.-O. Rous- 
set, B. Geyer, P.-L. Gatier et N. Awad, 153-168. Travaux de la Maison de l' Orient 71. Conquête de la steppe IV. Lyon: Maison de l' Orient et de la Méditerranée, 2016.

El-Faiz, Mohammed. Les Maîtres de l'eau: histoire de l'hydraulique arabe. Arles: Actes Sud, 2005.

Feissel, Denis, en collaboration avec Pierre-Louis Gatier. "Bulletin épigraphique: Syrie, Phénicie, Palestine, Arabie." Revue des Études grecques 116 (2003): 662-675.

Gatier, Pierre-Louis. "Une frontière sans limes?" Dans Aux origines de l'archéologie aérienne: A. Poidebard (1878-1955), édité par Lévon Nordiguian et Jean-François Salles, 139-149. Beyrouth: Université Saint-Joseph, 2000.

Gatier, Pierre-Louis. “«Grande» ou «petite Syrie Seconde»? Pour une géographie historique de la Syrie intérieure protobyzantine." Dans Conquête de la steppe et appropriation des terres sur les marges du croissant fertile, édité par Bernard Geyer, 91-109. Lyon: Maison de l' Orient et de la Méditerranée, 2001.

Gatier, Pierre-Louis. “Une borne d'Andrôna." Dans Habitat et environnement: prospections dans les Marges arides de la Syrie du Nord, édité par M.-O. Rousset, B. Geyer, P.-L. Gatier et N. Awad, 137-151. Travaux de la Maison de l'Orient 71. Conquête de la steppe IV. Lyon: Maison de l' Orient et de la Méditerranée, 2016.

Gatier, Pierre-Louis, Bernard Geyer et Marie-Odile Rousset, éds, Entre nomades et sédentaires: prospections en Syrie du Nord et en Jordanie du Sud. Lyon: Maison de l' Orient et de la Méditerranée, 2010.

Gaube, Heinz. "Die syrischen Wüstenschlösser: einige wirtschaftliche und politische Gesichtspunkte zu ihrer Entstehung." Zeitschrift des Deutschen Palästina-Vereins 95 (1979): 195-209.

Genequand, Denis. "Économie de production, affirmation du pouvoir et dolce vita: aspects de la politique de l' eau sous les Omeyyades au Bilad al-Sham." Dans Stratégies d'acquisition de l'eau et société au Moyen-Orient depuis l'Antiquité, édité par Mohamed al-Dbiyat et Michel Mouton, 157-177. Beyrouth: Presses de l'Ifpo, 2009.

Genequand, Denis. Les établissements des élites omeyyades en Palmyrène et au ProcheOrient. Bibliothèque Archéologique et Historique 200. Beyrouth: Presses de l'Ifpo, 2012.

Genequand, Denis, et Marie-Odile Rousset. "Résidences aristocratiques byzantines et omeyyades des marges arides du nord de la Syrie." Dans Habitat et environnement: prospections dans les Marges arides de la Syrie du Nord, édité par M.-O. Rousset, B. Geyer, P.-L. Gatier et N. Awad, 207-265. Travaux de la Maison de l'Orient 71. Conquête de la steppe IV. Lyon: Maison de l'Orient et de la Méditerranée, 2016.

Gentelle, Pierre. "Éléments pour une histoire des paysage et du peuplement du Djebel Hauran septentrional, en Syrie du Sud.” Dans Hauran I: Recherches archéologiques sur la Syrie du Sud à l'époque hellénistique et romaine, vol. 1, édité par Jean-Marie Dentzer, 19-62. Paris: Geuthner, 1985. 
Geyer, Bernard. "Des fermes byzantines aux palais omayyades ou l'ingénieuse mise en valeur des plaines steppiques de Chalcidique (Syrie)." Dans Aux origines de l'archéologie aérienne: A. Poidebard (1878-1955), édité par Lévon Nordiguian et JeanFrançois Salles, 109-122. Beyrouth: Université Saint-Joseph, 2000.

Geyer, Bernard, éd. Conquête de la steppe et appropriation des terres sur les marges du Croissant fertile. Lyon: Maison de l' Orient et de la Méditerranée, 2001.

Geyer, Bernard. "Pratiques d' acquisition de l' eau et modalités de peuplement dans les Marges arides de la Syrie du Nord." Dans Stratégies d'acquisition de l'eau et société au Moyen-Orient depuis l'Antiquité, édité par Mohamed al-Dbiyat et Michel Mouton, 25-43. Beyrouth: Presses de l'Ifpo, 2009.

Geyer, Bernard, et Marie-Odile Rousset. "Les steppes arides de la Syrie du Nord à l' époque byzantine ou la «ruée vers l'est»." Dans Conquête de la steppe et appropriation des terres sur les marges du croissant fertile, édité par Bernard Geyer, 115-118. Lyon: Maison de l' Orient et de la Méditerranée, 2001.

Geyer, Bernard, et Marie-Odile Rousset. "Rasm Abū Miyāl: château du désert." Dans Une aventure archéologique: Antoine Poidebard, photographe et aviateur, édité par Fabrice Denise et Lévon Nordiguian, 294-295. Marseille: Parenthèses, 2004.

Geyer, Bernard, et Marie-Odile Rousset. "Déterminants géoarchéologiques du peuplement rural dans les Marges arides de Syrie du Nord aux VII ${ }^{\mathrm{e}}-\mathrm{IX}^{\mathrm{e}}$ siècles." Dans $L e$ Proche-Orient deJustinien aux Abbassides:peuplement et dynamiques spatiales, édité par Antoine Borrut, Muriel Debié, Arietta Papaconstantinou, Dominique Pieri et Jean-Pierre Sodini, 77-92 et 369-374. Turnhout: Brepols, 2011.

Geyer, Bernard, Jacques Besançon et Marie-Odile Rousset. "Les peuplements anciens." Dans Les marges arides du croissant fertile, édité par Ronald Jaubert et Bernard Geyer, 55-69. Lyon: Maison de l' Orient et de la Méditerranée, 2006.

Geyer, Bernard, Marie-Odile Rousset et Jacques Besançon. "Les citernes pluviales des steppes syriennes, éléments de la conquête d' une marge aride." Dans Habitat et environnement: prospections dans les Marges arides de la Syrie du Nord, édité par M.O. Rousset, B. Geyer, P.-L. Gatier et N. Awad, 45-88. Travaux de la Maison de l' Orient 71. Conquête de la steppe IV. Lyon: Maison de l'Orient et de la Méditerranée, 2016.

Geyer, Bernard, Nazir Awad et Jacques Besançon. "Murs, murets et enclos: l'usage de la pierre dans la mise en valeur des steppes arides de Syrie du Nord." Dans De l'île d'Aphrodite au Paradis perdu, itinéraire d'un gentilhomme lyonnais: en hommage à Yves Calvet, édité par B. Geyer, V. Matoïan et M. Al-Maqdissi, 275-298. Ras ShamraOugarit XXII. Louvain: Peeters, 2015.

Griesheimer, Marc. "L'occupation byzantine sur les marges orientales du territoire d'Apamée de Syrie (d'après les inscriptions de Taroutia empôron et d'Androna)." Dans Conquête de la steppe et appropriation des terres sur les marges du croissant fertile, édité par Bernard Geyer, 123-144. Lyon: Maison de l'Orient et de la Méditerranée, 2001. 
Gogräfe, Rudiger. "Der Tempel von Isriye zwischen nahötlicher Kulttradition und römischer Architektur." Topoi 7, no. 2 (1997): 801-836.

Haase, Claus-Peter. "Ein archäologischer Survey im Gabal Sbet und im Gabal al-Ahass." Damaszener Mitteilungen 1 (1983): 69-76.

Hamidé, Abdul-Rahman. La région d'Alep:étude de géographie rurale. Paris: Imprimerie de l'Université, 1959.

Honigmann, Ernst. “Syria.” Dans Paulys Realencyclopädie der Classischen Alterumswissenschaft 4A, 2, édité par Georg Wissowa, Karl Mittelhaus et Wilhelm Kroll, col. 1549-727. Stuttgart:J.B. Metzler, 1932.

Jalabert, Louis, et René Mouterde. Inscriptions grecques et latines de la Syrie, vol. 2, Chalcidique et Antiochène. Paris: Geuthner, 1939.

Jalabert, Louis, René Mouterde et Claude Mondésert. Inscriptions grecques et latines de la Syrie, vol. 4, Laodicée, Apamène. Paris: Geuthner, 1955.

Jaubert, Ronald, Mohamed al-Dbiyat et Françoise Debaine. "Transformations des régions cultivées: différenciation des exploitations et extension de l'irrigation." Dans Les marges arides du Croissant fertile: peuplements, exploitation et contrôle des ressources en Syrie du Nord, édité par Ronald Jaubert et Bernard Geyer, 167-187. Lyon: Maison de l' Orient et de la Méditerrannée, 2006.

Jaubert, Ronald, Françoise Debaine, Jacques Besançon, Mohamed al-Dbiyat, Bernard Geyer, Gustave Gintzburger et Myriam Traboulsi, éds. Land Use and Vegetation Cover in the Semi-arid and Arid Areas of Aleppo and Hama Provinces (Syria). Lyon: Maison de l'Orient et de la Méditerranée, 1999.

Jaubert, Ronald, et Bernard Geyer, éds. Les marges arides du Croissant fertile: peuplements, exploitation et contrôle des ressources en Syrie du Nord. Lyon: Maison de l'Orient et de la Méditerranée, 2006.

Jaubert, Ronald, Bernard Geyer, Françoise Debaine et Mohamed Al-Dbiyat. “L' exploitation des eaux de subsurface des marges arides de Syrie: dégradation ou transformation?" Sécheresse 13, no. 1 (2002): 43-50.

Kaplan, Michel. Les hommes et la terre à Byzance du vie au XI ${ }^{e}$ siècle. Paris: Publications de la Sorbonne, 1992.

Kaplan, Michel. "L'économie paysanne dans l'Empire byzantin du ve au $\mathrm{X}^{\mathrm{e}}$ siècle." Dans Byzance: villes et campagnes, édité par Michel Kaplan, 31-65. Paris: Picard, 2006.

Kennedy, Hugh. "The Impact of Muslim Rule on the Pattern of Rural Settlement in Syria." Dans La Syrie de Byzance à l'Islam VII ${ }^{e}-V I I I^{e}$ siècles: actes du Colloque International, Lyon-Paris, 11-15 septembre 1990, édité par Pierre Canivet et Jean-Paul ReyCoquais, 291-297. Damas: Institut Français d'Études Arabes de Damas, 1992.

Kramers, Johannes Hendrik, et Farhad Daftary. "Salamiyya." Dans Encyclopaedia of Islam, 2nd edition, vol. 8. Édité par P. Bearman, Th. Bianquis, C.E. Bosworth, E. van Donzel, W.P. Heinrichs, 952-955. Leiden: Brill, 1995. 
Landry, Patrick. "Eaux souterraines et qanats d' après un livre arabe du XI ${ }^{\mathrm{e}}$ siècle." Dans Techniques et pratiques hydro-agricoles traditionnelles en domaine irrigué, approche pluridisciplinaire des modes de culture avant la motorisation en Syrie: Actes du colloque de Damas, 27 Juin-1 ${ }^{\text {er }}$ Juillet 1987, édité par Bernard Geyer, 271-283. Paris: Geuthner, 199 o.

Lassus, Jean. Inventaire archéologique de la région au nord-est de Hama. 2 vols. Damas: Institut français et Paris: Leroux, 1935-1936.

Latron, André. La vie rurale en Syrie et au Liban: étude d'économie sociale. Beyrouth: Imprimerie catholique, 1936.

Métral, Jean. "Économie et sociétés: stratégies alternatives et cultures de l'aléatoire." Dans Steppes d'Arabie: états, pasteurs, agriculteurs et commerçants: le devenir des zones sèches, édité par Riccardo Bocco, Ronald Jaubert et Françoise Métral, 381-387. Paris: Presses universitaires de France et Genève: Cahiers de l'I.U.E.D.

Miquel, André. La géographie humaine du monde musulman jusqu'au milieu du n ${ }^{e}$ siècle. Paris: École des Hautes Études en Sciences Sociales, 1980.

Morony, Michael. "Economic boundaries? Late Antiquity and Early Islam." Journal of the Economic and Social History of the Orient 47, no. 2 (2004): 166-194.

Morrisson, Cécile. "Le monnayage omeyyade et l'histoire administrative et économique de la Syrie." Dans La Syrie de Byzance à l'Islam VII ${ }^{e-V I I I}{ }^{e}$ siècles: Actes du Colloque International, Lyon-Paris, 11-15 septembre 1990, édité par Pierre Canivet et JeanPaul Rey-Coquais, 309-318. Damas: Institut Français d'Études Arabes de Damas, 1992.

Mouterde, René, et Antoine Poidebard. Le Limes de Chalcis: organisation de la steppe en Haute-Syrie romaine. 2 vols. Paris: Geuthner, 1945.

Mundell Mango, Marlia. "Excavations and Survey at Androna, Syria: The Oxford Team 1999." Dumbarton Oaks Papers 56 (2002): 307-315.

Mundell Mango, Marlia. "Fishing in the Desert." Dans Golden Gate, Festchrift for Ihor Ševcenko, édité par Peter Schneider et Olga Strakhov, 323-33o. Palaeoslavica 10, no. 1 (2002).

Mundell Mango, Marlia. "Baths, Reservoirs and Water Use at Androna in Late Antiquity and the Early Islamic Period.” Dans Residences, Castles, Settlements:Transformation Processes from Late Antiquity to Early Islam in Bilad al-Sham, édité par Karin Bartl et Abd al-Razzaq Moaz, 73-88. Damas: Deustches Archäologisches Institut, 2008.

Musil, Alois. Palmyrena: A Topographical Itinerary. New York: AMs Press, 1928.

Rigot, Jean-Baptiste. "Environnement naturel et occupation du sol dans le bassinversant du lac Jabbûl (Syrie du Nord) à l' Holocène." PhD diss., Université LumièreLyon 2, 2003.

Rivoal, Marion. "Le peuplement byzantin et la mise en valeur de la Syrie centrale: l' exemple des plateaux basaltiques (Jebel al-Ala, Jebel Hass et Jebel Shbayt)." Dans Regards croisés sur l'étude archéologique des paysages anciens: nouvelles recherches 
dans le Bassin méditerranée, en Asie centrale et au Proche et Moyen-Orient, édité par Hala Alarashi, Marie-Laure Chambrade, Sébastion Gondet, Aurélie Jouvenel, Caroline Sauvage et Hervé Tronchère, 79-92. Lyon: Maison de l'Orient et de la Méditerranée, 2010.

Rivoal, Marion. "Le peuplement byzantin des massifs basaltiques de la Syrie centrale: modalités d'occupation du sol et stratégies de mise en valeur." Dans Entre nomades et sédentaires: prospections en Syrie du Nord et en Jordanie du Sud, édité par PierreLouis Gatier, Bernard Geyer et Marie-Odile Rousset, 209-240. Lyon: Maison de l' Orient et de la Méditerranée, 2010.

Rivoal, Marion. “Entre steppe et plateaux basaltiques: l' occupation de la Syrie centrale à la période byzantine." Syria 88 (2011): 111-121.

Rivoal, Marion. "Autour des dépotoirs: production, gestion et exploitation des excréments animaux dans la steppe syrienne pendant l' Antiquité tardive." Dans Habitat et environnement: prospections dans les Marges arides de la Syrie du Nord, édité par M.-O. Rousset, B. Geyer, P.-L. Gatier et N. Awad, 169-201. Travaux de la Maison de l' Orient 71. Conquête de la steppe IV. Lyon: Maison de l' Orient et de la Méditerranée, 2016.

Robert, Jeanne et Louis. "Bulletin épigraphique." Revue des Études Grecques (19461947): 298-372.

Robert, Jeanne et Louis. "Bulletin épigraphique." Revue des Études Grecques (1954): 95193 .

Rousset, Marie-Odile. "Qanats de la steppe syrienne." Dans Entre nomades et sédentaires: prospections en Syrie du Nord et en Jordanie du Sud, édité par Pierre-Louis Gatier, Bernard Geyer et Marie-Odile Rousset, 241-270. Lyon: Maison de l'Orient et de la Méditerranée, 2010.

Rousset, Marie-Odile. "Le peuplement de la steppe de Syrie du Nord entre le II $^{\mathrm{e}}$ s. av. et le IV s. apr. J.-C." Syria 88 (2011): 123-139.

Rousset, Marie-Odile. "De Chalcis à Qinnasrin." Dans Topoï: villes et campagnes aux rives de la Méditerranée ancienne: hommages à Georges Tate, édité par G. Charpentier et V. Puech, 311-340. Supplément 12 (2013).

Rousset, Marie-Odile. "Traces of the Banu Salih in the Syrian Steppe? The Fortresses of Qinnasrin and Abu al-Khanadiq." Levant 45 (2013), 69-95.

Rousset, Marie-Odile. "Deux sites fortifiés au début de l'époque islamique au Bilād al-Šām: Qinnasrīn et Abū al-Hुanādiq." Dans La guerre dans le Proche-Orient médiéval $\left(X^{e}-X V^{e}\right.$ s. $)$ : etat de la question, lieux communs, nouvelles approches, édité par Mathieu Eychenne, Stéphane Pradines et Abbès Zouache, 193-229. Recherches d' archéologie, de philologie et d' histoire 37. Le Caire: IFAO, 2014.

Rousset, Marie-Odile, Bernard Geyer, Pierre-Louis Gatier et Nazir Awad. Habitat et environnement: prospections dans les marges arides de la Syrie du Nord. Conquête de la steppe. Lyon: Maison de l'Orient et de la Méditerranée, 2016. 
Rousset, Marie-Odile, et Catherine Duvette. "L'élevage dans la steppe à l' époque byzantine: indices archéologiques." Dans Les villages dans l'Empire byzantin, $I V^{e}-X V^{e}$ siècle, édité par Jean Lefort, Cécile Morrisson et Jean-Pierre Sodini, 485-494. Paris: Lethielleux, 2005.

Tchalenko, Georges. Villages antiques de la Syrie du Nord. Le massif du Bélus à l'époque romaine. 3 vols. Paris: Geuthner, 1953-1958.

Traboulsi, Myriam. "Les précipitations dans les Marges arides de la Syrie du Nord." Dans Entre nomades et sédentaires: prospections en Syrie du Nord et en Jordanie du Sud, édité par Pierre-Louis Gatier, Bernard Geyer et Marie-Odile Rousset, 73-108. Lyon: Maison de l' Orient et de la Méditerranée, 2010.

Villeneuve, François. "L'économie rurale et la vie des campagnes dans le Hauran antique ( $\mathrm{I}^{\mathrm{er}}$ s. av. J.-C.-VII ${ }^{\mathrm{e}}$ s. ap. J.-C.): une approche." Dans Hauran I: recherches archéologiques sur la Syrie du Sud à l'époque hellénistique et romaine, vol. 1, édité par Jean-Marie Dentzer, 63-129. Paris: Geuthner, 1985

Wessels, Joshka. "Tunneling for survival: ICARDA, Aleppo (Syria)." ICARDA Caravan 11 (1999): 12-13.

Wessels, Joshka. "Reviving Ancient Water Tunnels in the Desert - Digging for Gold?" Journal of Mountain Science 2, no. 4 (2005): 294-305.

Weulersse, Jacques. Paysans de Syrie et du Proche-Orient. Paris: Gallimard, 1946.

Zakri, Simone. "Pratiques hydroagricoles traditionnelles d' après les manuscrits arabes d' agronomie à la période médiévale dans le monde arabo-musulman." Dans Techniques et pratiques hydro-agricoles traditionnelles en domaine irrigué, approche pluridisciplinaire des modes de culture avant la motorisation en Syrie: Actes du colloque de Damas, 27 Juin-1 ${ }^{\text {er }}$ Juillet 1987, édité par Bernard Geyer, 35-51. Paris: Geuthner, 199o.

Zuckerman, Constantin. "Comtes et ducs en Égypte autour de l' an 400 et la date de la Notitia Dignitatum Orientis." Antiquité Tardive 6 (1998): 137-147. 